\title{
Hyperfine interactions and internal rotation in methanol
}

Boy Lankhaar, Gerrit C. Groenenboom, and Ad van der Avoird

Citation: J. Chem. Phys. 145, 244301 (2016); doi: 10.1063/1.4972004

View online: http://dx.doi.org/10.1063/1.4972004

View Table of Contents: http://aip.scitation.org/toc/jcp/145/24

Published by the American Institute of Physics 


\title{
Hyperfine interactions and internal rotation in methanol
}

\author{
Boy Lankhaar, ${ }^{1,2}$ Gerrit C. Groenenboom,${ }^{1}$ and Ad van der Avoird ${ }^{1, a)}$ \\ ${ }^{1}$ Theoretical Chemistry, Institute for Molecules and Materials, Radboud University, Heyendaalseweg 135 , \\ 6525 AJ Nijmegen, The Netherlands \\ ${ }^{2}$ Department of Earth and Space Sciences, Chalmers University of Technology, Onsala Space Observatory, \\ 43992 Onsala, Sweden
}

(Received 10 November 2016; accepted 28 November 2016; published online 22 December 2016)

\begin{abstract}
We present a rigorous derivation of the nuclear spin-rotation and spin-torsion coupling terms in the hyperfine Hamiltonian for molecules with internal rotation. Our formulas differ from the expressions derived by Heuvel and Dymanus [J. Mol. Spectrosc. 47, 363 (1973)], which these authors used and which were also applied recently by others to interpret experimental hyperfine spectra of such molecules. In the present work, our theoretical results are applied to methanol. We calculate the nuclear spin-spin magnetic dipole-dipole interactions and the nuclear contribution to the spin-torsion coupling vectors from the nuclear coordinates as functions of the internal rotation angle $\gamma$, compute the spin-rotation coupling tensors by ab initio electronic structure methods also as functions of $\gamma$, and obtain the missing parameters for the electronic contribution to the spin-torsion coupling from a fit to measured spectra. The resulting hyperfine Hamiltonian is then used to compute hyperfine transition frequencies and intensities for twelve torsion-rotation transitions in methanol. With the use of the $a b$ initio calculated spin-rotation coupling parameters without any modification, and physically reasonable values for the spin-torsion coupling parameters from the fit, we find good agreement with all of the measured spectra. Published by AIP Publishing. [http://dx.doi.org/10.1063/1.4972004]
\end{abstract}

\section{INTRODUCTION}

Radiation emitted from star-forming regions by molecular masers such as water, $\mathrm{OH}$, and methanol is used by radio-astronomers to extract information on magnetic fields in different sections of the accretion disk around the protostar. ${ }^{1-3}$ The relevant molecular parameters to relate the observed Zeeman shifts between left and right circularly polarized radiation to the local magnetic field strength are well known for $\mathrm{H}_{2} \mathrm{O}$ and $\mathrm{OH}$, but not for methanol. Detailed knowledge of the hyperfine splitting of the torsion-rotation levels of methanol is required, but cannot be extracted from the available experimental data alone. Methanol has also been identified as the most sensitive molecule for a search of a varying proton-electron mass ratio on a cosmological time scale. ${ }^{4}$ Observations of extragalactic methanol have led to a constraint on the variation of this ratio. ${ }^{5-7}$ The line shapes in the observed spectra may be affected by underlying hyperfine structure, but this has not been included in the analysis. In the studies of galactic cold cores the lines are narrower, which makes hyperfine structure an essential ingredient to include in analyses.

The theoretical description of hyperfine coupling in molecules with internal rotation such as methanol has been addressed in some papers cited below. The present paper revisits the theory, derives a slightly but significantly different formalism, and applies it to determine the hyperfine structure of methanol in different torsion-rotation states. Just as the recent study on methanol by Coudert et al., ${ }^{8}$ we combine $a b$ initio electronic structure calculations and fits to experimental data to obtain the relevant hyperfine coupling parameters.

a)A.vanderAvoird@theochem.ru.nl
The dominant hyperfine interactions in closed-shell molecules are the magnetic dipole-dipole coupling between the nuclei with spin $I>0$, the electric quadrupole coupling for nuclei with spin $I \geq 1$, and the interaction of the nuclear spins with the magnetic field generated by overall rotation of the molecule, the so-called spin-rotation coupling. The theoretical description of all these interactions is well known. In molecules with internal rotation, such as methanol, also the internal rotation generates a magnetic field and an additional interaction term occurs: spin-torsion coupling. A theoretical description of spin-torsion coupling was given in 1972 by Heuvel and Dymanus ${ }^{9,10}$ who also performed measurements on methanol ${ }^{9,11}$ and applied their theory to extract the relevant coupling parameters from the experimental data. The symmetry properties of the torsion-rotation Hamiltonian including these hyperfine couplings were discussed by Hougen et al. ${ }^{12}$ Further measurements on the hyperfine structure of methanol were reported in 2015 by Coudert et al.; ${ }^{8}$ the interpretation of their experimental data was based on the theory of Ref. 10 . The parameters fitted in Ref. 8 to the measured hyperfine spectra of methanol showed some peculiar features; however: (i) the dominant spin-rotation coupling parameter from the fit deviates much more from the value obtained by the advanced electronic structure calculations than could be expected on the basis of results for other molecules, and (ii) the dominant spintorsion coupling parameter computed with a formula for the nuclear contribution from Ref. 10 was increased by a factor of 2 in the fit, instead of showing the expected reduction by the electronic contribution. These discrepancies could be due to some inconsistencies in the formulas of Ref. 10, especially regarding the way to apply the so-called Thomas precession correction. 
Thomas precession is a purely kinematic effect that follows from the special theory of relativity, but does not vanish at small velocities. It is named after Thomas who showed ${ }^{13}$ in 1927 that the correction factor associated with this precession is essential to obtain the correct mathematical expression for the spin-orbit coupling in atoms. For nuclear spin-rotation coupling in molecules, the precession is related to the acceleration of the nuclei in the molecule into curved paths by the electrostatic forces from the electrons and the other nuclei. Such a Thomas precession correction is included in the formulas of Heuvel and Dymanus, ${ }^{10}$ but the correction factor in that paper, and also in the earlier paper on spin-rotation coupling by Gunther-Mohr, Townes, and van Vleck ${ }^{14}$ is not correct. ${ }^{15}$ It was given correctly in Ref. 16, but the precession correction was omitted entirely in the formula for spin-rotation coupling in Flygare's textbook. ${ }^{17}$ The omission of the Thomas precession correction from his book is justified because he had derived ${ }^{15}$ in 1964 that this correction completely vanishes when the nuclei in the molecule are in their equilibrium positions, where they do not experience any net force. When the molecule vibrates about its equilibrium geometry, the Thomas precession correction does not vanish, and it has actually been computed for several diatomic molecules ${ }^{18,19}$ from a formula derived in Refs. 19 and 20. The amplitudes of vibration in these systems are small; however, and the Thomas precession correction has only a minute effect on the calculated spin-rotation coupling constants. For molecules with large amplitude internal motions, such as the torsional motion in methanol, one would think that it might be more substantial. Here, we derive a formula for the Thomas precession correction associated with the internal rotation in molecules and apply it to methanol.

Another combined experimental and theoretical study of the hyperfine structure in the torsion-rotation spectrum of methanol was performed by Belov et al. ${ }^{21}$ They investigated transitions between torsion-rotation levels of $E$ symmetry with angular momenta $J$ ranging from 13 to 34 by Lamb-dip submillimeter-wave spectroscopy and found unexpectedly large doublet splittings in the spectra due to hyperfine coupling. These doublet splittings occur for several, but not for all transitions, and they were explained by a theoretical model involving torsionally mediated spin-rotation coupling with parameters fitted to the experimental data. In Sec. IV B we will briefly refer to these results in relation to our results obtained with the full hyperfine Hamiltonian for a rotational transition between the levels with $J=8$ and 9.

The starting point in our study of methanol is its torsionrotation states. They can be derived from the Hamiltonian given by Hougen et al. ${ }^{22}$ It is based on the classical expression for the kinetic energy of a molecule with internal rotation about a single axis derived by Lin and Swalen. ${ }^{23}$ Hougen et al. express this Hamiltonian in different coordinate systems and show that its eigenstates are most easily evaluated with the so-called rho-axis method (RAM). Their paper also includes a discussion of the symmetry properties of the Hamiltonian. A program, BELGI, ${ }^{22}$ was developed to carry out the evaluation of the torsion-rotation levels. The parameters in the Hamiltonian were obtained by $\mathrm{Xu}$ et al. ${ }^{24}$ from a global fit of these levels to a large data set of observed spectral transitions. To supplement this Hamiltonian with the correct expressions for hyperfine interactions, we briefly recapitulate and then extend its derivation, while paying special attention to the form of the operators representing spin-rotation and spin-torsion coupling. We also evaluated the nuclear spin magnetic dipole-dipole interactions and carried out electronic structure calculations with the program package CFOUR, ${ }^{25}$ to obtain numerical values for the spin-rotation coupling tensors in methanol as functions of the internal rotation angle. Calculation of the spintorsion coupling tensors is not implemented in this program, so we applied our formula for spin-torsion coupling to evaluate the nuclear contribution to these tensors and we estimate the electronic contributions from a fit of our calculated spectra to experimental data. ${ }^{8,11}$ Furthermore, we computed the Thomas precession correction to the spin-rotation and spintorsion tensors with the formula that we derived for molecules with internal rotation. Finally, we used our hyperfine Hamiltonian with the $a b$ initio calculated and fitted parameters to compute the hyperfine levels for several rotational states of methanol, generated transition frequencies and intensities, and compared our results to the measured spectra.

\section{THEORY}

\section{A. Hyperfine coupling}

Methanol, $\mathrm{CH}_{3} \mathrm{OH}$, is an asymmetric rotor molecule, consisting of a symmetric rotor "top," the $\mathrm{CH}_{3}$ group, attached to a "frame," the $\mathrm{OH}$ group. The internal rotation of the methyl group with respect to the hydroxyl group is hindered by a potential $V(\gamma)$ depending on the internal rotation angle $\gamma$ with a threefold barrier of $374 \mathrm{~cm}^{-1} .{ }^{24}$ For the lowest energy levels, the internal rotation or torsion is classically forbidden, but occurs by quantum mechanical tunneling. The carbon and oxygen nuclei in their most abundant ${ }^{12} \mathrm{C}$ and ${ }^{16} \mathrm{O}$ forms have nuclear spin $I=0$. The only nuclei involved in hyperfine coupling are the four hydrogen nuclei with spin $I=1 / 2$. The hyperfine coupling Hamiltonian of a molecule with internal rotation is given by

$$
H^{\text {hyper }}=H^{\mathrm{DD}}+H^{\mathrm{SR}}+H^{\mathrm{ST}},
$$

where

$$
H^{\mathrm{DD}}=\sum_{K<L} \hat{\boldsymbol{I}}_{K} \cdot \boldsymbol{D}_{K L}(\gamma) \hat{\boldsymbol{I}}_{L}
$$

describes the magnetic dipole-dipole coupling between the nuclei, $H^{\mathrm{SR}}$ is the nuclear spin-rotation coupling, and $H^{\mathrm{ST}}$ is the spin-torsion coupling. The nuclei are labeled with $K$ and $L$, and $\hat{\boldsymbol{I}}_{K}$ are the nuclear spin operators. The form of the magnetic dipole-dipole coupling tensor $\boldsymbol{D}_{K L}$, a second rank irreducible tensor, is well known. It is also valid for molecules with internal rotation, where it depends on the angle $\gamma$.

The expressions for the spin-rotation and spin-torsion coupling terms in the Hamiltonian for a molecule with internal rotation

$$
\begin{aligned}
H^{\mathrm{SR}}= & -\sum_{K} \hat{\boldsymbol{I}}_{K} \cdot \boldsymbol{M}_{K}(\gamma) \hat{\boldsymbol{J}} \\
& +\sum_{K} 2 F \hat{\boldsymbol{I}}_{K} \cdot \boldsymbol{M}_{K}(\gamma) \boldsymbol{I}^{\mathrm{top}} \boldsymbol{\lambda}\left(\hat{p}_{\gamma}-\boldsymbol{\rho} \cdot \hat{\boldsymbol{J}}\right)
\end{aligned}
$$

and

$$
H^{\mathrm{ST}}=-\sum_{K} 2 F \hat{\boldsymbol{I}}_{K} \cdot \boldsymbol{w}_{K}(\gamma)\left(\hat{p}_{\gamma}-\boldsymbol{\rho} \cdot \hat{\boldsymbol{J}}\right)
$$


are derived in Appendix A. The spin-rotation coupling tensors are given by $\boldsymbol{M}_{K}(\gamma)=\boldsymbol{W}_{K}(\gamma) \boldsymbol{I}^{-1}$ and the tensors $\boldsymbol{W}_{K}(\gamma)$, as well as the spin-torsion coupling vectors $\boldsymbol{w}_{K}(\gamma)$ are also defined in Appendix A. The total inertia tensor $\boldsymbol{I}$ does not depend on $\gamma$ because the rotating "top" with inertia tensor $\boldsymbol{I}^{\text {top }}$ is a symmetric rotor. The unit vector $\lambda$ is the direction of the internal rotation axis; the constant $F$ and the constant vector $\boldsymbol{\rho}=\boldsymbol{I}^{-1} \boldsymbol{I}^{\mathrm{top}} \boldsymbol{\lambda}$ are defined in Eq. (A8). All tensor and vector components are given with respect to the principal axis frame MF of the whole molecule, in which the total inertia tensor $\boldsymbol{I}$ is diagonal. The operator $\hat{\boldsymbol{J}}$ represents the total rotational angular momentum, $\hat{p}_{\gamma}=(\hbar / i) \partial / \partial \gamma$ is the torsional angular momentum operator, and $\hbar$ is the reduced Planck constant.

The derivation in Appendix A starts by recalling the derivation of the torsion-rotation kinetic energy operator, since this operator is needed to obtain the correct expressions for the total angular momentum $\hat{\boldsymbol{J}}$ and the torsional angular momentum $\hat{p}_{\gamma}$. In the formulas in Eq. (A6) for their classical equivalents, one can observe that for molecules with internal rotation not only the overall angular velocity $\omega$ appears in the total angular momentum $\boldsymbol{J}$ but also the torsional velocity $\dot{\gamma}$. Viceversa, not only $\dot{\gamma}$ appears in the torsional angular momentum $p_{\gamma}$ but also $\omega$. By consequence, we find that the expression for the spin-rotation coupling Hamiltonian $H^{\mathrm{SR}}$ in Eq. (3) not only contains the usual terms $-\hat{\boldsymbol{I}}_{K} \cdot \boldsymbol{M}_{K} \hat{\boldsymbol{J}}$ but also the contributions that involve both the torsional and overall angular momenta $\hat{p}_{\gamma}$ and $\hat{\boldsymbol{J}}$. Vice-versa, the spin-torsion coupling Hamiltonian in Eq. (4) involves not only the angular momentum $\hat{p}_{\gamma}$ but also the rotational angular momentum $\hat{\boldsymbol{J}}$.

The additional terms in the spin-rotation Hamiltonian have a form that is similar to the spin-torsion Hamiltonian and can be absorbed into the latter by defining

$$
\boldsymbol{w}_{K}^{\prime}(\gamma)=\boldsymbol{w}_{K}(\gamma)-\boldsymbol{M}_{K}(\gamma) \boldsymbol{I}^{\mathrm{top}} \boldsymbol{\lambda}
$$

The total spin-rotation-torsion Hamiltonian then becomes

$$
H^{\mathrm{SR}}+H^{\mathrm{ST}}=-\sum_{K}\left[\hat{\boldsymbol{I}}_{K} \cdot \boldsymbol{M}_{K}(\gamma) \hat{\boldsymbol{J}}+2 F \hat{\boldsymbol{I}}_{K} \cdot \boldsymbol{w}_{K}^{\prime}(\gamma)\left(\hat{p}_{\gamma}-\boldsymbol{\rho} \cdot \hat{\boldsymbol{J}}\right)\right] .
$$

In methanol the unit vector $\lambda$ in the direction of the internal rotation axis is very nearly parallel to the principal axis $a$ of the $\mathrm{CH}_{3} \mathrm{OH}$ molecule and we find that

$$
I^{\mathrm{top}} \lambda \approx I_{\gamma} \lambda,
$$

where $I_{\gamma}=\boldsymbol{\lambda} \cdot \boldsymbol{I}^{\mathrm{top}} \boldsymbol{\lambda}$ is the moment of inertia of the "top" rotating about the axis $\lambda$. Equation (6) can then be rewritten as

$H^{\mathrm{SR}}+H^{\mathrm{ST}}=-\sum_{K}\left[\hat{\boldsymbol{I}}_{K} \cdot \boldsymbol{M}_{K}(\gamma) \hat{\boldsymbol{J}}+f \hat{\boldsymbol{I}}_{K} \cdot \boldsymbol{d}_{K}^{\prime}(\gamma)\left(\hat{p}_{\gamma}-\boldsymbol{\rho} \cdot \hat{\boldsymbol{J}}\right)\right]$

with the dimensionless factor

$$
f=2 F I_{\gamma}=(1-\rho \cdot \lambda)^{-1} .
$$

By analogy with Eq. (5) we obtain

$$
\boldsymbol{d}_{K}^{\prime}(\gamma)=\boldsymbol{d}_{K}(\gamma)-\boldsymbol{M}_{K}(\gamma) \lambda
$$

with the spin-torsion coupling vector

$$
\boldsymbol{d}_{K}(\gamma)=\boldsymbol{w}_{K}(\gamma) I_{\gamma}^{-1}
$$

The operators $\hat{\boldsymbol{I}}_{K}$ and $\hat{\boldsymbol{J}}$ operate on the spin and spatial coordinates of the nuclei, respectively, so they commute. Their Cartesian components are Hermitian operators and the Cartesian components of the tensors $\boldsymbol{M}_{K}$ and the vectors $\boldsymbol{w}_{K}^{\prime}$ and $\boldsymbol{d}_{K}^{\prime}$ are real-valued. Hence, the Hamiltonians in Eqs. (6) and (8) are Hermitian operators, even though the spin-rotation coupling tensors $\boldsymbol{M}_{K}$ are not symmetric. We recall that both the tensors $\boldsymbol{M}_{K}$ and the spin-torsion coupling vectors $\boldsymbol{d}_{K}$ depend on the internal rotation angle $\gamma$. Furthermore, we recall that the components of the rotational angular momentum operator $\hat{\boldsymbol{J}}$ with respect to the MF frame obey anomalous commutation relations. ${ }^{26}$

\section{B. Thomas precession correction}

In our derivations, we assume that both the "frame" and the "top" parts in a molecule with internal rotation are rigid and that the nuclei in each part are fixed at their equilibrium positions. A formula for the Thomas precession correction for diatomic molecules vibrating around their equilibrium structure has been derived in Refs. 19 and 20, by an extension of Flygare's derivation ${ }^{15}$ showing that this correction vanishes for molecules at their equilibrium geometry. When the molecule is not at its equilibrium geometry, the Thomas precession correction is directly related to the forces on the nuclei.

The result for diatomic molecules in Refs. 19 and 20 can also be derived directly by starting from the textbook formula ${ }^{27}$ for the Thomas precession correction to the energy of a particle $K$ (in this case, a nucleus) with spin $\boldsymbol{I}_{K}$ moving in a magnetic field

$$
U_{T}^{(K)}=\boldsymbol{I}_{K} \cdot \boldsymbol{\omega}_{T}^{(K)} .
$$

The Thomas precession frequency depends on the acceleration $\boldsymbol{a}_{K}$ and velocity $\boldsymbol{v}_{K}$ of the particle and (for velocities much smaller than the speed of light $c$ ) is given by

$$
\boldsymbol{\omega}_{T}^{(K)}=\frac{1}{2 c^{2}} \boldsymbol{a}_{K} \times \boldsymbol{v}_{K}
$$

The acceleration of a nucleus in a molecule is determined by the net force $\boldsymbol{F}_{K}$ acting on it; it vanishes when the molecule is in its equilibrium geometry. When it is not, the forces on the nuclei in a diatomic molecule simply follow from the derivative of the intramolecular potential with respect to the internuclear distance. By relating the acceleration $\boldsymbol{a}_{K}$ in the above formula to this derivative, one easily obtains the result of Refs. 19 and 20 .

In molecules with weakly hindered internal rotation one must consider large deviations from their equilibrium geometry. Methanol, for instance, shows a large amplitude internal rotation of the $\mathrm{CH}_{3}$ "top" with respect to the $\mathrm{OH}$ "frame." In our MF system of axes the "frame" stays at rest, while the "top" rotates. The acceleration of the hydrogen nuclei in the "top" is given by

$$
\boldsymbol{a}_{K}=\frac{\boldsymbol{F}_{K}}{m_{K}}=-\frac{1}{m_{K}\left|\boldsymbol{\sigma}_{K}\right|} \frac{d V(\gamma)}{d \gamma} \boldsymbol{s}_{K},
$$

where $\left|\sigma_{K}\right|$ is the distance of nucleus $K$ to the internal rotation axis, the torsional potential $V(\gamma)$ depends on the internal rotation angle $\gamma, \hat{\sigma}_{K}$ is a unit vector in the direction of the vector $\sigma_{K}$, and the unit vector $s_{K}=\lambda \times \hat{\sigma}_{K}$ points in the direction of the motion of nucleus $K$. The velocity of a hydrogen nucleus $K$ 
in the rotating "top" depends on the internal rotation velocity $\dot{\gamma}$, as well as on the overall rotational velocity $\omega$,

$$
\boldsymbol{v}_{K}=\omega \times \boldsymbol{r}_{K}+\dot{\gamma} \lambda \times \boldsymbol{r}_{K} .
$$

The $\dot{\gamma}$ term does not contribute to the Thomas precession as it points in the same direction as the acceleration $\boldsymbol{a}_{K}$. Substitution of these results into Eq. (13) yields

$$
\begin{aligned}
\boldsymbol{\omega}_{T}^{(K)} & =-\frac{1}{2 m_{K}\left|\boldsymbol{\sigma}_{K}\right| c^{2}} \frac{d V(\gamma)}{d \gamma} \boldsymbol{s}_{K} \times\left(\boldsymbol{\omega} \times \boldsymbol{r}_{K}\right) \\
& =\frac{1}{2 m_{K}\left|\boldsymbol{\sigma}_{K}\right| c^{2}} \frac{d V(\gamma)}{d \gamma}\left[\left(\boldsymbol{r}_{K} \cdot \boldsymbol{s}_{K}\right) \mathbf{1}-\boldsymbol{r}_{K} \otimes \boldsymbol{s}_{K}\right] \boldsymbol{\omega} .
\end{aligned}
$$

The symbol 1 represents the $3 \times 3$ unit matrix, while $\otimes$ stands for the tensor product of two vectors. The angular velocity $\omega$ is related to the total angular momentum $\boldsymbol{J}$ and the torsional angular momentum $p_{\gamma}$ by Eq. (A7), and the Thomas correction to the energy is given in operator form by

$$
U_{T}^{(K)}=\hat{\boldsymbol{I}}_{K} \cdot \boldsymbol{Q}_{K} \hat{\boldsymbol{J}}-2 F \hat{\boldsymbol{I}}_{K} \cdot \boldsymbol{q}_{K}\left(\hat{p}_{\gamma}-\boldsymbol{\rho} \cdot \hat{\boldsymbol{J}}\right)
$$

with

$$
\boldsymbol{Q}_{K}=\frac{1}{2 m_{K}\left|\boldsymbol{\sigma}_{K}\right| c^{2}} \frac{d V(\gamma)}{d \gamma}\left[\left(\boldsymbol{r}_{K} \cdot \boldsymbol{s}_{K}\right) \mathbf{1}-\boldsymbol{r}_{K} \otimes \boldsymbol{s}_{K}\right] \boldsymbol{I}^{-1}
$$

and

$$
\boldsymbol{q}_{K}=\frac{1}{2 m_{K}\left|\boldsymbol{\sigma}_{K}\right| c^{2}} \frac{d V(\gamma)}{d \gamma}\left[\left(\boldsymbol{r}_{K} \cdot \boldsymbol{s}_{K}\right) \mathbf{1}-\boldsymbol{r}_{K} \otimes \boldsymbol{s}_{K}\right] \boldsymbol{\rho} .
$$

The tensors $\boldsymbol{Q}_{K}$ and vectors $\boldsymbol{q}_{K}$ may be considered as the Thomas precession corrections to the spin-rotation coupling tensors $\boldsymbol{M}_{K}$ and spin-torsion coupling vectors $\boldsymbol{w}_{K}^{\prime}$ in Eq. (6). For methanol, we obtained Eq. (8), and $\boldsymbol{q}_{K} I_{\gamma}^{-1}$ is the correction to $\boldsymbol{d}_{K}^{\prime}$. Both $\boldsymbol{Q}_{K}$ and $\boldsymbol{q}_{K}$ depend on the torsion angle $\gamma$ and they can be directly computed from the nuclear positions in the molecule and the derivative $d V / d \gamma$ of the known potential $V(\gamma)$.

\section{Symmetry}

For molecules with internal motions that have multiple equilibrium geometries, i.e., multiple equivalent minima on their potential surface, one must use the permutation-inversion (PI) group or molecular symmetry group ${ }^{28,29}$ rather than the point group of a single equilibrium structure. We label the $\mathrm{CH}_{3}$ protons in methanol with $1,2,3$ and the $\mathrm{OH}$ proton with 4. Internal rotation of the $\mathrm{CH}_{3}$ group corresponds to the cyclic permutations (123) and (132). The permutationinversion operation $(23)^{*}$, i.e., the interchange of protons 2 and 3 combined with inversion corresponds to reflection in the point group $C_{3 v}$ of the equilibrium structure. The PI group $G_{6}$ of internally rotating methanol, isomorphic to $C_{3 v}$, is generated by (123) and (23)* It has two one-dimensional irreducible representations (irreps), $A_{1}$ and $A_{2}$, and a two-dimensional one, $E$. The application of $G_{6}$ to molecules with internal rotation similar to methanol has extensively been discussed by Hougen et al. ${ }^{22}$ They show, in particular, how the generators (123) and $(23)^{*}$ act on the torsion-rotation wave functions $\psi_{J, K_{a}}^{v_{\tau}, \sigma}$,

$$
\begin{aligned}
& (123) \psi_{J, K_{a}}^{v_{\tau}, \sigma}=\exp (2 \pi \sigma i / 3) \psi_{J, K_{a}}^{v_{\tau}, \sigma} \\
& (23)^{*} \psi_{J, K_{a}}^{v_{\tau}, \sigma}=(-1)^{J-K_{a}} \psi_{J,-K_{a}}^{v_{\tau},-\sigma} .
\end{aligned}
$$

The quantum number $J$ is the total angular momentum, $K_{a}$ is its projection on the $a$ axis of the MF frame, $v_{\tau}$ is the torsional quantum number and $\sigma$ is the torsional symmetry quantum number, adopting the values 0 and \pm 1 . It follows from these equations that functions with $\sigma=0$ transform according to the $A_{1}$ or $A_{2}$ irreps, and functions with $\sigma= \pm 1$ span the $E$ irrep.

When investigating hyperfine splittings of the torsionrotation levels, one must also include the nuclear spin wave functions of the four protons. This is most conveniently done by first coupling the spins $I_{1}, I_{2}, I_{3}$ of the equivalent protons in the $\mathrm{CH}_{3}$ group. This yields one set of functions with total spin $I_{123}=3 / 2$ with projections $M_{I}=-3 / 2,-1 / 2,1 / 2,3 / 2$, and two sets of functions with $I_{123}=1 / 2$ and $M_{I}=-1 / 2,1 / 2$. Since, inversion does not affect the nuclear spin functions, it follows from their behavior under the permutations (123) and (23) that the functions with $I_{123}=3 / 2$ span the irrep $A_{1}$ of $G_{6}$, while the two sets of functions with $I_{123}=1 / 2$ each carry the irrep $E$. Next, these functions are coupled with the nuclear spin $I_{4}=1 / 2$ of the $\mathrm{OH}$ proton, which yields functions $\left|\left(I_{123}, I_{4}\right) I\right\rangle$ of $A_{1}$ symmetry with total spin $I=1$ and 2 , and functions of $E$ symmetry with total spin $I=0$ and 1 .

Since protons are fermions, the Pauli principle requires that the total torsion-rotation-spin wave functions are antisymmetric under odd permutations and symmetric under even permutations. The Pauli principle does not impose any conditions on the inversion behavior of the wave function, so total wave functions of $A_{1}$ and $A_{2}$ symmetry in the group $G_{6}$ are both appropriate. One way to obtain such total wave functions is by taking the product of a nuclear spin wave function $\left|\left(I_{123}=3 / 2, I_{4}\right) I\right\rangle$ of $A_{1}$ symmetry and a torsion-rotation wave function with $\sigma=0$,

$$
\left[\psi_{J, K_{a}}^{v_{\tau}, 0} \pm(-1)^{J-K_{a}} \psi_{J,-K_{a}}^{v_{\tau}, 0}\right] / \sqrt{2},
$$

that has $A_{1}$ or $A_{2}$ symmetry. Another way is by combining two nuclear spin wave functions $\left|\left(I_{123}=1 / 2, I_{4}\right) I\right\rangle$ that together carry irrep $E$ with torsion-rotation wave functions with $\sigma= \pm 1$ that also carry irrep $E$. If we set up a basis $\left\{\psi_{1}^{E}, \psi_{2}^{E}\right\}$ $=\left\{\psi_{J, K_{a}}^{v_{\tau}, 1},(-1)^{J-K_{a}} \psi_{J,-K_{a}}^{v_{\tau},-1}\right\}$, the operations (123) and (23)* are represented by the irrep matrices

$\boldsymbol{P}_{123}^{E}=\left(\begin{array}{cc}e^{2 \pi i / 3} & 0 \\ 0 & e^{-2 \pi i / 3}\end{array}\right) \quad$ and $\quad \boldsymbol{P}_{23^{*}}^{E}=\left(\begin{array}{ll}0 & 1 \\ 1 & 0\end{array}\right)$.

By taking appropriate linear combinations of the wave functions $\left|\left(I_{123}=1 / 2, I_{4}\right) I\right\rangle$, one can construct nuclear spin wave functions $\left\{\phi_{1}^{E}, \phi_{2}^{E}\right\}$ that carry the same $E$ irrep matrix $\boldsymbol{P}_{23^{*}}$ and a matrix complex conjugate to $\boldsymbol{P}_{123}$. Total wave functions of $A_{1}$ and $A_{2}$ symmetry obeying the Pauli principle are then obtained by taking

$$
\Psi^{\mathrm{tot}}=\left[\psi_{1}^{E} \phi_{1}^{E} \pm \psi_{2}^{E} \phi_{2}^{E}\right] / \sqrt{2}
$$

\section{METHODS}

\section{A. Torsion-rotation structure}

The internal-rotation Hamiltonian used to calculate the torsion-rotation levels and wave functions is defined by the rho axis method (RAM) described in Refs. 22 and 30-32. The $z$-axis of the RAM frame is parallel to the vector $\rho$ defined in Eq. (A8), which has the advantage that only the term $2 F \rho \hat{p}_{\gamma} \hat{J}_{z}$ 
in the Hamiltonian of Eq. (A9) couples the internal and overall rotation, so that all operators containing the torsional angular momentum $\hat{p}_{\gamma}$ are diagonal in the rotational quantum number $K_{a}$. The operator $\hat{J}_{z}$ with eigenvalues $K_{a}$ is the projection of the total angular momentum $\hat{\boldsymbol{J}}$ on the $z$-axis of the RAM frame, $\rho$ is the length of the vector $\rho$, and $F$ is defined in Eq. (A8). In methanol, the angle between the vector $\rho$, i.e., the $z$-axis of the RAM frame, and the principal $a$ axis is less than half a degree. We take this $a$ axis as the $z$-axis of our principal axes frame MF and assume that the RAM frame coincides with our MF frame in this case.

The eigenstates of this Hamiltonian are determined in two steps. In the first step, the torsional Hamiltonian

$$
\hat{H}^{\text {tors }}=F\left(\hat{p}_{\gamma}-\rho \hat{J}_{z}\right)^{2}+V(\gamma)
$$

is diagonalized for each value of $K_{a}$ in a basis consisting of functions $\exp [i(3 k+\sigma) \gamma] / \sqrt{2 \pi}$, with $k$ running from -10 to 10 . The eigenfunctions from this first step are the torsional wave functions: $\left|\left(K_{a}\right) v_{\tau} \sigma\right\rangle$. In the second step, these torsional wave functions for all $2 J+1$ values of $K_{a}$ are included in the calculation of the rotational states for given $J$. They are multiplied with the symmetric rotor functions $\left|J M K_{a}\right\rangle=\sqrt{\frac{2 J+1}{8 \pi^{2}}} D_{M K_{a}}^{(J)}(\chi, \theta, \phi)^{*}$ that depend on the overall rotation angles $(\chi, \theta, \phi)$ to provide the basis in which the full torsion-rotation Hamiltonian is diagonalized. This procedure is implemented in the BELGI code. ${ }^{22}$ The standard version of the code was modified and improved by Xu et al. ${ }^{24}$ who fitted a set of 119 molecular parameters of methanol to a dataset of 25000 measured spectral transitions and reproduced the lower torsion-rotation energy levels with an accuracy better than $100 \mathrm{kHz} .{ }^{4}$ The resulting parameters are listed in Table 2 of Ref. 24.

\section{B. Hyperfine levels}

\section{Hyperfine coupling tensors}

Spin-rotation coupling tensors $\boldsymbol{M}_{K}(\gamma)$ can be obtained from $a b$ initio electronic structure calculations with the program package CFOUR. ${ }^{25}$ Calculations with CFOUR were carried out at the coupled-cluster level of theory including single and double excitations with perturbative addition of the triples contribution $[\mathrm{CCSD}(\mathrm{T})]$, in an augmented triple-zeta correlation-consistent (aug-cc-pVTZ) basis set. ${ }^{33}$ The geometry of methanol was optimized at this level, which yields $\mathrm{OH}$ and $\mathrm{CO}$ bond lengths of 0.956 and $1.427 \AA$, respectively, a $\mathrm{COH}$ bond angle of $108.87^{\circ}$ and a torsional $\mathrm{HOCH}$ angle of $180^{\circ}$. Averaging the equilibrium values to maintain $C_{3 v}$ symmetry of the rotating $\mathrm{CH}_{3}$ group, we find $\mathrm{CH}$ bond lengths and OCH bond angles of $1.096 \AA$ and $109.91^{\circ}$.

The electronic contributions to the spin-rotation coupling tensors $\boldsymbol{M}_{K}(\gamma)$ were calculated at the same level of theory for 13 equidistant values of the torsional angle $\gamma$ by keeping the HOC fragment fixed and rotating the $\mathrm{CH}_{3}$ group over these angles about the $\mathrm{OC}$ bond axis. The nuclear contributions to the tensors $\boldsymbol{M}_{K}(\gamma)$ were also given by CFOUR, but were also calculated directly from the nuclear coordinates. Also the magnetic dipole-dipole coupling tensors $\boldsymbol{D}_{K L}(\gamma)$ were calculated from the nuclear coordinates for the same 13 values of $\gamma$, as well as the nuclear contributions to the spin-torsion coupling vectors $\boldsymbol{d}_{K}(\gamma)$. For the latter, we used Eqs. (11) and (A17). Calculation of the electronic contribution to the spin-torsion vectors $\boldsymbol{d}_{K}(\gamma)$ is not implemented in CFOUR. We obtained it from a fit to measured hyperfine spectra described below. Results that we computed for other, rigid, molecules such as $\mathrm{H}_{2} \mathrm{O}$ showed us that spin-rotation tensors $\boldsymbol{M}_{K}(\gamma)$ from electronic structure calculations at this level of theory are quite accurate, so we kept our ab initio values for these tensors. Still, in contrast with the work of Coudert et al. ${ }^{8}$ who included these values in their fit, we could obtain a good fit of the experimental data by fitting only the spin-torsion vectors $\boldsymbol{d}_{K}(\gamma)$. All individual elements of the tensors $\boldsymbol{M}_{K}(\gamma)$ and $\boldsymbol{D}_{K L}(\gamma)$ and the vectors $\boldsymbol{d}_{K}(\gamma)$ were expanded in a Fourier series in $\gamma$. Some of the elements are symmetric with respect to a sign change of $\gamma$ and were expanded in functions $\cos n \gamma$, others are antisymmetric and were expanded in functions $\sin n \gamma$.

\section{Thomas precession corrections}

In Sec. II B we recalled the result from the literature that the Thomas precession correction to the spin-rotation coupling vanishes for molecules at their equilibrium geometry. For molecules with a large amplitude internal rotation, such as methanol, we derived Eq. (18) for the Thomas correction $\boldsymbol{Q}_{K}$ to the spin-rotation coupling tensors and Eq. (19) for the Thomas correction $\boldsymbol{q}_{K}$ to the spin-torsion coupling vectors. The correction involves only the protons in the rotating $\mathrm{CH}_{3}$ "top" and it requires knowledge of the torsional potential $V(\gamma)$. For methanol this potential can be written as

$$
V(\gamma)=\sum_{n=1}^{3} \frac{V_{3 n}}{2}(1-\cos 3 n \gamma)
$$

The parameters $V_{3}, V_{6}, V_{9}$ in this expression are known from the work by $\mathrm{Xu}$ et al. ${ }^{24}$ The parameters $V_{6}$ and $V_{9}$ are very small, so they will be neglected here. The height of the threefold barrier, $V_{3}$, is $374 \mathrm{~cm}^{-1}$.

\section{Matrix elements and hyperfine levels}

With the knowledge of all coupling tensors $\boldsymbol{D}_{K L}(\gamma)$, $\boldsymbol{M}_{K}(\gamma)$, and $\boldsymbol{d}_{K}(\gamma)$ in the hyperfine Hamiltonian, we can compute the hyperfine levels. The Hamiltonian is diagonalized in the basis $\left|\left\{\left(I_{123}, I_{4}\right) I, J\right\} F M_{F}\right\rangle$ obtained by coupling the eigenfunctions of the torsion-rotation problem described in Sec. III A with the nuclear spin functions $\left|\left(I_{123}, I_{4}\right) I\right\rangle$ defined in Sec. II C. Coupling the total nuclear spin $I$ with the torsion-rotation angular momentum $J$ yields the total angular momentum $F$ and its projection $M_{F}$ on the space-fixed $z$-axis. The torsion-rotation wave functions have quantum numbers $v_{\tau}, J, K_{a}$ and symmetry $A$ or $E$, the nuclear spin basis of the same symmetry has $I_{123}=3 / 2$ or $1 / 2$, see Sec. II C. The rotational quantum number $K_{a}$ is only an approximate one, a rotational wave function with given $J, K_{a}$ actually contains basis functions with all $K_{a}$ ranging from $-J$ to $J$. Also the torsional and rotational quantum numbers $v_{\tau}$ and $J$ are approximate ones, but the energy gaps between the torsion-rotation states are typically on the order of a few $\mathrm{GHz}$, while the hyperfine coupling terms in methanol amount to about $10 \mathrm{kHz}$. Hence, the hyperfine Hamiltonian will hardly mix basis functions with different $v_{\tau}$ and $J$ and we may restrict our basis to a 
single value of these quantum numbers. With this assumption, the spin-rotation and spin-torsion Hamiltonian in Eq. (8) can be rewritten ${ }^{12,34-36}$ as

$$
\hat{H}_{K}^{\mathrm{SR}}+\hat{H}_{K}^{\mathrm{ST}}=\left(\hat{O}_{K}^{\mathrm{SR}}+\hat{O}_{K}^{\mathrm{ST}}\right)\left(\hat{\boldsymbol{I}}_{K}^{\mathrm{SF}} \cdot \hat{\boldsymbol{J}}^{\mathrm{SF}}\right) .
$$

The advantage of this factorization is that the scalar product operator $\hat{\boldsymbol{I}}_{K} \cdot \hat{\boldsymbol{J}}$ is invariant under rotation, which we used to express it in terms of the space-fixed (SF) components of the operators. Matrix elements over the SF basis $\left|\left\{\left(I_{123}, I_{4}\right) I, J\right\} F M_{F}\right\rangle$ are thus more easily evaluated. The operators

$$
\hat{O}_{K}^{\mathrm{SR}}=\frac{1}{2 J(J+1)} \hat{\boldsymbol{J}} \cdot \boldsymbol{M}_{K}(\gamma) \hat{\boldsymbol{J}}+\text { hermitian conjugate }
$$

and

$$
\begin{aligned}
\hat{O}_{K}^{\mathrm{ST}}= & \frac{1}{2 J(J+1)} f \hat{\boldsymbol{J}} \cdot \boldsymbol{d}_{K}^{\prime}(\gamma)\left(\hat{p}_{\gamma}-\boldsymbol{\rho} \cdot \hat{\boldsymbol{J}}\right) \\
& + \text { hermitian conjugate }
\end{aligned}
$$

contain the body-fixed components of $\hat{\boldsymbol{J}}$ with respect to the MF frame, and also the tensors $\boldsymbol{M}_{K}(\gamma)$ and the vectors $\boldsymbol{d}_{K}^{\prime}(\gamma)$ are given with respect to this frame. Matrix elements of the operators $\hat{O}_{K}^{\mathrm{SR}}$ and $\hat{O}_{K}^{\mathrm{ST}}$ over the torsion-rotation eigenfunctions are simply scalar values.

Also the dipole-dipole coupling Hamiltonian can be factorized into a part with body-fixed and a part with only space-fixed angular momentum operators

$$
\hat{H}_{K L}^{\mathrm{DD}}=\hat{O}_{K L}^{\mathrm{DD}}\left[\frac{3}{2}\left(\hat{\boldsymbol{I}}_{K}^{\mathrm{SF}}+\hat{\boldsymbol{I}}_{L}^{\mathrm{SF}}\right) \cdot \hat{\boldsymbol{J}}^{\mathrm{SF}}-\left(\hat{\boldsymbol{I}}_{K}^{\mathrm{SF}} \cdot \hat{\boldsymbol{I}}_{L}^{\mathrm{SF}}\right)\left(\hat{\boldsymbol{J}}^{\mathrm{SF}}\right)^{2}\right],
$$

where

$$
\hat{O}_{K L}^{\mathrm{DD}}=\frac{2}{J(J+1)(J-1)(2 J+3)} \hat{\boldsymbol{J}} \cdot \boldsymbol{D}_{K L}(\gamma) \hat{\boldsymbol{J}} .
$$

Also the matrix elements of $\hat{O}_{K L}^{\mathrm{DD}}$ are computed in the $\mathrm{MF}$ frame. Diagonalization of the matrix of the total hyperfine Hamiltonian in Eq. (1) for given $v_{\tau}$ and $J$ yields a set of hyperfine levels labeled with the quantum number $F$.

\section{Intensities}

In order to compare our results with measured hyperfine spectra, we also computed the line strengths of the transitions between hyperfine levels. The dipole moment of methanol was calculated $a b$ initio as a function of the internal rotation angle $\gamma$ with the finite-field method at the same level of theory and with the same basis as the hyperfine coupling tensors. The spherical components of the dipole in the SF frame are related to the components calculated in the MF frame as $\mu_{m}^{\mathrm{SF}}=\sum_{q} D_{m q}^{(1) *}(\chi, \theta, \phi) \mu_{q}^{\mathrm{MF}}$, with $(\chi, \theta, \phi)$ being the overall rotation angles. Matrix elements of the SF dipole components $\mu_{m}$ over the coupled basis $\left|\left\{\left(I_{123}, I_{4}\right) I, J\right\} F M_{F}\right\rangle$ needed to compute rotational and hyperfine transition dipole moments were evaluated with the spherical tensor techniques explained in Appendix B.

\section{RESULTS}

\section{A. Calculated results}

The $a b$ initio calculated results for the spin-rotation coupling tensors $\boldsymbol{M}_{K}(\gamma)$ and the calculated and fitted results

TABLE I. Coefficients $a_{n}$ in the Fourier expansion describing the $\gamma$ dependence of the spin-rotation coupling tensors $\boldsymbol{M}_{K}(\gamma)$ calculated $a b$ initio. The components

\begin{tabular}{|c|c|c|c|c|c|c|c|c|c|}
\hline & Symmetry & $a_{0}$ & $a_{1}$ & $a_{2}$ & $a_{3}$ & $a_{4}$ & $a_{5}$ & $a_{6}$ & $a_{0}^{\mathrm{Th}}\left(\approx a_{6}^{\mathrm{Th}}\right)$ \\
\hline$M_{a a}^{\mathrm{CH} 3}$ & + & 12.486 & 0.741 & -0.080 & 0.052 & -0.005 & $5.33 \cdot 10^{-4}$ & $2.23 \cdot 10^{-4}$ & $-1.40 \cdot 10^{-8}$ \\
\hline$M_{b b}^{\mathrm{CH} 3}$ & + & 0.579 & -0.104 & -1.660 & -0.018 & 0.002 & $-9.08 \cdot 10^{-4}$ & $-1.45 \cdot 10^{-4}$ & $1.3 \cdot 10^{-5}$ \\
\hline$M_{c c}^{\mathrm{CH} 3}$ & + & 0.688 & -0.065 & 1.673 & 0.011 & -0.003 & 0.001 & $1.42 \cdot 10^{-4}$ & $-1.2 \cdot 10^{-5}$ \\
\hline$M_{a b}^{\mathrm{CH} 3}$ & + & 0.731 & -4.430 & 0.578 & -0.131 & 0.022 & -0.002 & $-3.13 \cdot 10^{-4}$ & $4.85 \cdot 10^{-8}$ \\
\hline$M_{b a}^{\mathrm{CH} 3}$ & + & 0.150 & -1.325 & 0.083 & -0.021 & -0.002 & $-4.36 \cdot 10^{-4}$ & $-5.73 \cdot 10^{-5}$ & $-3.6 \cdot 10^{-6}$ \\
\hline$M_{a c}^{\mathrm{CH} 3}$ & - & 0 & 4.690 & -0.605 & 0.128 & -0.024 & 0.002 & $1.16 \cdot 10^{-4}$ & 0 \\
\hline$M_{c a}^{\mathrm{CH} 3}$ & - & 0 & 1.342 & -0.086 & 0.019 & 0.003 & $4.02 \cdot 10^{-4}$ & $1.06 \cdot 10^{-4}$ & 0 \\
\hline$M_{b c}^{\mathrm{CH} 3}$ & - & 0 & $5.38 \cdot 10^{-5}$ & 1.721 & 0.013 & -0.002 & 0.001 & 0 & 0 \\
\hline$M_{c b}^{\mathrm{CH} 3}$ & - & 0 & 0.055 & 1.614 & 0.016 & -0.003 & 0.001 & $1.43 \cdot 10^{-4}$ & 0 \\
\hline$M_{a a}^{\mathrm{OH}}$ & + & 13.305 & 0 & 0 & -0.380 & 0 & 0 & -0.001 & 0 \\
\hline$M_{b b}^{\mathrm{OH}}$ & + & 0.659 & 0 & 0 & 0.004 & 0 & 0 & $1.71 \cdot 10^{-4}$ & 0 \\
\hline$M_{c c}^{\mathrm{OH}}$ & + & 2.935 & 0 & 0 & -0.048 & 0 & 0 & $-1.26 \cdot 10^{-4}$ & 0 \\
\hline$M_{a b}^{\mathrm{OH}}$ & + & 6.139 & 0 & 0 & -0.048 & 0 & 0 & $-1.79 \cdot 10^{-4}$ & 0 \\
\hline$M_{b a}^{\mathrm{OH}}$ & + & 1.461 & 0 & 0 & -0.032 & 0 & 0 & $-3.98 \cdot 10^{-4}$ & 0 \\
\hline$M_{a c}^{\mathrm{OH}}$ & - & 0 & 0 & 0 & 0.002 & 0 & 0 & $-4.03 \cdot 10^{-4}$ & 0 \\
\hline$M_{c a}^{\mathrm{OH}}$ & - & 0 & 0 & 0 & 0.067 & 0 & 0 & $5.24 \cdot 10^{-4}$ & 0 \\
\hline$M_{b c}^{\mathrm{OH}}$ & - & 0 & 0 & 0 & -0.049 & 0 & 0 & $-2.45 \cdot 10^{-4}$ & 0 \\
\hline$M_{c b}^{\mathrm{OH}}$ & - & 0 & 0 & 0 & 0.002 & 0 & 0 & $4.72 \cdot 10^{-5}$ & 0 \\
\hline
\end{tabular}
of $\boldsymbol{M}_{K}$ are defined with respect to the principal axes $a, b$, and $c$. Terms in the Fourier series are $a_{n} \cos n \gamma$ or $a_{n} \sin n \gamma$, depending on whether a component is symmetric (+) or antisymmetric (-) under the PI operation $(23)^{*}$ that involves a sign change of the torsional angle $\gamma$. The superscripts $\mathrm{CH}_{3}$ and $\mathrm{OH}_{\text {refer to the }}$ $\mathrm{CH}_{3}$ protons $(K=1,2,3)$ and $\mathrm{OH}$ proton $(K=4)$, respectively. Results are given only for the $\mathrm{CH}_{3}$ proton with $K=1$, the values for $K=2,3$ correspond to a change of $\gamma$ by $120^{\circ}$ and $240^{\circ}$. All spin-rotation coupling expansion coefficients $a_{n}$ and the coefficients $a_{n}^{\text {Th }}$ of the Thomas precession corrections $Q_{K}$ are given in $\mathrm{kHz}$. 
TABLE II. Coefficients $a_{n}^{\text {nuc }}$ in the Fourier expansion describing the $\gamma$ dependence of the nuclear contribution to the spin-torsion coupling vectors $\boldsymbol{d}_{K}(\gamma)$ calculated from the nuclear coordinates. The four coefficients $a_{0}^{\text {el }}$ of the $\gamma$-independent electronic contribution, as well as one $a_{1}^{\mathrm{el}}$ coefficient, are obtained from a fit to measured hyperfine spectra, see text. The components of $\boldsymbol{d}_{K}$ are defined with respect to the principal axes $a, b$, and $c$. Their $\gamma$ dependence is expanded in functions $\cos n \gamma$ terms or $\sin n \gamma$ for + and - symmetry, respectively. The superscripts $\mathrm{CH}_{3}$ and $\mathrm{OH}$ refer to the $\mathrm{CH}_{3}$ protons $(K=1,2,3)$ and $\mathrm{OH}$ proton $(K=4)$, respectively. Results are given only for the $\mathrm{CH}_{3}$ proton with $K=1$, the values for $K=2,3$ correspond to a change of $\gamma$ by $120^{\circ}$ and $240^{\circ}$. All spin-torsion coupling expansion coefficients $a_{n}$ and the coefficients $a_{n}^{\text {Th }}$ of the Thomas precession corrections $\boldsymbol{q}_{K} / I_{\gamma}$ are given in $\mathrm{kHz}$.

\begin{tabular}{lcccccccc}
\hline \hline & Symmetry & $a_{0}^{\text {nuc }}$ & $a_{1}^{\text {nuc }}$ & $a_{3}^{\text {nuc }}$ & $a_{6}^{\text {nuc }}$ & $a_{0}^{\text {el }}$ & $a_{1}^{\text {el }}$ & $a_{0}^{\mathrm{Th}}\left(\approx a_{6}^{\mathrm{Th}}\right)$ \\
\hline$d_{a}^{\mathrm{CH} 3}$ & + & 80.00 & 0.319 & $1.40 \cdot 10^{-5}$ & 0 & -66.41 & -2.76 & $-1.13 \cdot 10^{-8}$ \\
$d_{b}^{\mathrm{CH} 3}$ & + & 4.435 & -9.549 & 0 & 0 & -10.16 & 0 & $-2.91 \cdot 10^{-6}$ \\
$d_{c}^{\mathrm{CH} 3}$ & - & 0 & 0 & -9.44 & 0 & 0 & 0 & 0 \\
\hline$d_{a}^{\mathrm{OH}}$ & + & 11.66 & 0 & $-6.79 \cdot 10^{-2}$ & $-3.45 \cdot 10^{-4}$ & -0.462 & 0 & 0 \\
$d_{b}^{\mathrm{OH}}$ & + & 21.48 & 0 & $-9.49 \cdot 10^{-2}$ & $4.78 \cdot 10^{-4}$ & -53.99 & 0 & 0 \\
$d_{c}^{\mathrm{OH}}$ & - & 0 & 0 & 0.122 & $6.06 \cdot 10^{-4}$ & 0 & 0 & 0 \\
\hline \hline
\end{tabular}

for the spin-torsion coupling vectors $\boldsymbol{d}_{K}(\gamma)$ are listed in Tables I and II, respectively. Also the values computed for the corresponding Thomas-precession corrections are given in these tables. We found that the Thomas corrections are several orders of magnitude smaller than the spin-rotation tensors and spin-torsion vectors, so they may be safely neglected. This is perhaps somewhat surprising because the internal rotation in methanol is a large-amplitude internal motion of the molecule far from equilibrium. One must realize, however, that also away from the equilibrium geometry the net force on the $\mathrm{CH}_{3}$ protons due to the torsional potential is relatively small (the barrier is only $374 \mathrm{~cm}^{-1}=0.0017$ hartree) in comparison with the Coulomb interactions between the individual nuclei and electrons. The ab initio calculated dipole moment vector is given in Table III. All tensor and vector components are defined in the principal axes frame MF.

\section{B. Fit and comparison with experiment}

The hyperfine structure in four rotational transitions of methanol was measured in 1973 by Heuvel and Dymanus. ${ }^{11}$ Hyperfine spectra for the same and several other rotational transitions were recently reported by Coudert et al. ${ }^{8}$ The spectra of Coudert et al. ${ }^{8}$ were obtained by Fourier-transform microwave spectroscopy and they basically show the same spectrum twice. The two spectra should be nearly identical, in principle, but they are separated by a constant frequency due to the Doppler shift between the absorption of the microwave beams that propagate parallel and antiparallel to the molecular beam. Table I in Ref. 8 gives an overview of the measured transitions. All transitions refer to the torsional ground

TABLE III. Coefficients $a_{n}$ in the Fourier expansion of the dipole vector, in units $e a_{0}$. Components are defined in the principal axis frame. Their $\gamma$ dependence is expanded in functions $\cos n \gamma$ or $\sin n \gamma$ for + and - symmetry, respectively.

\begin{tabular}{ccccc}
\hline \hline & Symmetry & $a_{0}$ & $a_{3}$ & $a_{6}$ \\
\hline$\mu_{a}$ & + & -0.3678 & 0.001706 & $5.392 \cdot 10^{-6}$ \\
$\mu_{b}$ & + & -0.5635 & -0.01171 & $2.665 \cdot 10^{-5}$ \\
$\mu_{c}$ & - & 0 & 0.01113 & $2.528 \cdot 10^{-5}$ \\
\hline \hline
\end{tabular}

state $\left(v_{\tau}=0\right)$ with $\sigma=0$ and \pm 1 for levels of $A$ and $E$ symmetry, respectively. The quantum number $K_{c}$ in the label of an $A$ state with given $J_{K a}$ may adopt two values, which correspond to rotational levels of $A_{1}$ and $A_{2}$ symmetry or, in other words, to the parity of the torsion-rotation states under $(23)^{*}$, cf. Eq. (21).

In our calculations of the hyperfine spectra, we address the same transitions. We find that each rotational transition actually splits into many more hyperfine lines than resolved in the measured spectra. Hence, we could not fit our unknown spintorsion coupling parameters directly to the measured spectra. The same problem was encountered in the fits of these spectra by Coudert et al. ${ }^{8}$ who determined only the largest two components of the spin-rotation coupling and spin-torsion coupling tensors for the $\mathrm{CH}_{3}$ and $\mathrm{OH}$ protons by fitting the gap between two broad peaks in the measured spectra, each corresponding to several hyperfine transitions. We follow the same procedure, but we kept all spin-rotation coupling parameters fixed at the $a b$ initio calculated values, as well as the nuclear contributions to the spin-torsion coupling vectors, while we fitted the electronic contributions to the $a$ and $b$ components of these vectors for the $\mathrm{CH}_{3}$ and $\mathrm{OH}$ protons. The spectra used in our fit correspond to the $5_{15} A_{2} \leftarrow 6_{06} A_{1}$ and $3_{2} \leftarrow 3_{1} E$ transitions, as they both are $\Delta K_{a} \neq 0$ transitions, which makes them sensitive to spin-torsion interaction. After exploratory investigations of the effects of the fit parameters on the calculated spectra, the torsion-independent contributions to the components $d_{a}^{\mathrm{CH}_{3}}, d_{b}^{\mathrm{CH}_{3}}, d_{a}^{\mathrm{OH}}$, and $d_{b}^{\mathrm{OH}}$ of the electronic spintorsion vectors were varied in the ranges of $[-77.5,-57.5]$, $[-34.05,25.95],[-6.3,0]$, and $[-75,-50] \mathrm{kHz}$, respectively, and the $\cos (\gamma)$ dependent contribution to $d_{a}^{\mathrm{CH}_{3}}$ in the range of $[-30,30] \mathrm{kHz}$. In the fit of the $5_{15} A_{2} \leftarrow 6_{06} A_{1}$ spectrum, we used the Doppler-shifted spectrum at lower frequency and in the fit of the $3_{2} \leftarrow 3_{1} E$ spectrum the higher frequency spectrum. The Gaussian and Lorentzian line width parameters $\sigma$ and $\gamma$ were estimated from the experimental spectra and kept constant in the fits. The fit was performed in two steps. First we roughly determined the minimum in the absolute deviation between our calculated and the measured spectra by making 50000 random searches in the parameter space defined by an equidistant grid in each of the ranges. Next, in order to refine 

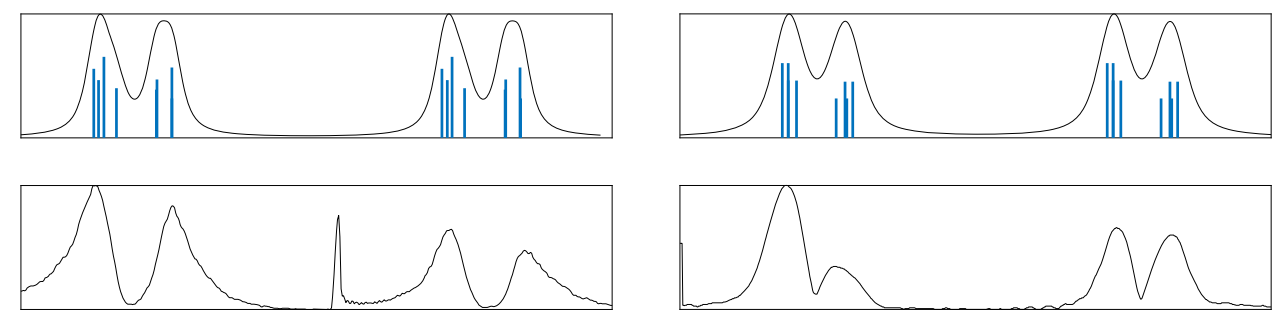

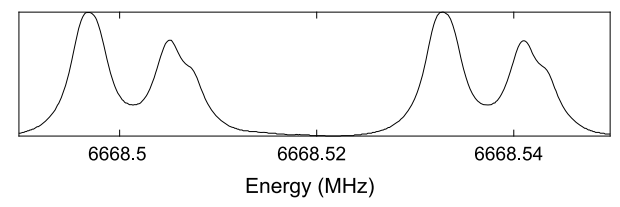

(a) $5_{15} A_{2} \leftarrow 6_{06} A_{1}$

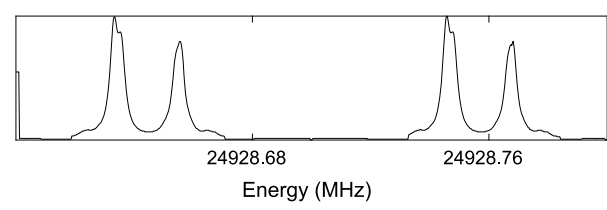

(b) $3_{2} E \leftarrow 3_{1} E$

FIG. 1. Comparison spectra calculated (upper) for the $A$ and $E$ levels with the measured spectra (middle) $)^{8,11}$ and the fit by Coudert $e t$ al. ${ }^{8}$ (bottom). The $5_{15} A_{2} \leftarrow 6_{06} A_{1}$ and $3_{2} E \leftarrow 3_{1} E$ transitions are observed by Coudert et al. ${ }^{8}$ with Doppler splittings of $35.37 \mathrm{kHz}$ and $109.89 \mathrm{kHz}$, respectively. The narrow peak in the center of the measured spectrum for the $5_{15} A_{2} \leftarrow 6_{06} A_{1}$ transition is an experimental artefact. ${ }^{8}$ The blue bars indicate the individual hyperfine transitions with a length proportional to the intensity. The hyperfine transitions are convoluted using a Voigt profile with Gaussian width $\sigma=0.5 \mathrm{kHz}$ and Lorentz width $\gamma=0.88 \mathrm{kHz}$ for the $5_{15} A_{2} \leftarrow 6_{06} A_{1}$ transition and $\sigma=2.5 \mathrm{kHz}$ and $\gamma=4.0 \mathrm{kHz}$ for the $3_{2} E \leftarrow 3_{1} E$ transition.

this minimum, we made 5000 search steps on a more restricted but finer grid around the minimum found in the first step.

The parameters for the electronic contributions to the spin-torsion interaction extracted from the fit are included in Table II. Figure 1 shows our fitted and measured spectra, as well as the spectra from the fit by Coudert et al. ${ }^{8}$ The figures also show our calculated line spectra (blue bars) with the heights of the lines given by the calculated relative intensities. Our fitted spectra are generated by the convolution of these line spectra with a Voigt profile.

The general form of each of the two Doppler components in both spectra is well reproduced. For the $5_{15} A_{2} \leftarrow 6_{06} A_{1}$ transition we find two peaks in each component, with the lowfrequency peak being slightly stronger, separated in frequency by a similar amount as the two peaks in the experimental spectrum. For the $3_{2} E \leftarrow 3_{1} E$ transition we also find two peaks, with the low-frequency peak also being slightly stronger, and again a frequency separation similar to experiment. The fit of Coudert $e t$ al. predicts this as well, although, in their case the individual hyperfine transitions seem to be more closely spaced which produces narrower peaks. In both experimental spectra, the two components of the Doppler doublet are different in intensity, while in the spectrum for the $3_{2} E \leftarrow 3_{1} E$ transition also the relative intensity of the two peaks differs between the two Doppler components.

The parameters in Table II show that the electronic contributions to the spin-torsion coupling vectors, all have a sign that is opposite to the sign of the corresponding ab initio calculated nuclear contributions, so they partly cancel each other. This is what one would expect, but it was not found in the fit of Coudert et al. ${ }^{8}$ where the electronic contributions had the same sign as the corresponding nuclear contributions and about the same magnitude. Moreover, we recall that we kept our $a b$ initio calculated values for the spin-rotation and magnetic dipole-dipole coupling tensors, i.e., we did not include those in our fit. Thus, we could fit all constant components of the electronic spin-torsion coupling vectors and part of their torsional dependence, whereas Coudert et al. fitted only the constant $a$-components of the coupling tensors. The fit values reveal interesting information, for instance, that the largest electronic contribution to the spin-torsion coupling vector $\boldsymbol{d}^{\mathrm{OH}}$ is the component along the $b$-axis. Table IV lists the expectation values of the operators $\hat{O}_{K}^{\mathrm{SR}}, \hat{O}_{K}^{\mathrm{ST}}$, and $\hat{O}_{K L}^{\mathrm{DD}}$ over the rotational states $J_{K_{q} K_{c}}^{v_{\tau}=0}$ of $A$ symmetry computed with our $a b$ initio calculated and fitted coupling parameters.

Spectra measured for other $A$ and $E$ symmetry transitions are simulated with the hyperfine levels and transition intensities computed with our ab initio calculated and fitted coupling parameters. Spectra for three $A$-symmetry transitions are shown in Fig. 2 and spectra for three of the $E$ symmetry transitions in Fig. 3. More transitions analyzed are given in Fig. 4 and in the supplementary material. Note that the spectra for the $A$ symmetry transitions in Fig. 2 were measured by Heuvel and Dymanus ${ }^{11}$ and the figures show only a single spectrum, while the spectra for the $E$ symmetry transitions in Fig. 3 were measured by microwave spectroscopy and the figures show the two Doppler-component spectra. In our discussion of the latter spectra, we refer only to the structure of a single Doppler component.

TABLE IV. Expectation values of the operators in Eqs. (27), (28), and (30) over torsion-rotation states of $A$ symmetry. We used the $a b$ initio data of Table I the fitted data from Table II and compute dipole-dipole interactions from the geometry. The subscripts $\mathrm{CH}_{3}$ and $\mathrm{OH}$ of the dipole-dipole coupling operator denote the coupling between two $\mathrm{CH}_{3}$ protons and the coupling between the $\mathrm{OH}$ proton and one of the $\mathrm{CH}_{3}$ protons, respectively.

\begin{tabular}{lrrrcrr}
\hline \hline$J_{K a K c}$ & $O_{1,2,3}^{\mathrm{SR}}$ & $O_{1,2,3}^{\mathrm{ST}}$ & $O_{4}^{\mathrm{SR}}$ & $O_{4}^{\mathrm{ST}}$ & \multicolumn{1}{c}{$O_{\mathrm{CH}_{3}}^{\mathrm{DD}}$} & \multicolumn{1}{c}{$O_{\mathrm{OH}}^{\mathrm{DD}}$} \\
\hline $1_{01}$ & 0.632 & -0.001 & 1.785 & -0.006 & -4.206 & 2.400 \\
$1_{11}$ & 6.602 & 4.078 & 7.950 & -7.960 & 2.094 & -1.113 \\
$1_{10}$ & 6.547 & 4.077 & 6.916 & -7.967 & 2.112 & -1.293 \\
$2_{12}$ & 2.640 & 1.353 & 4.185 & -2.693 & -0.503 & 0.308 \\
$2_{11}$ & 2.585 & 1.352 & 3.150 & -2.700 & -0.499 & 0.265 \\
$3_{13}$ & 1.650 & 0.671 & 3.245 & -1.376 & -0.351 & 0.210 \\
$3_{12}$ & 1.595 & 0.670 & 2.210 & -1.383 & -0.349 & 0.191 \\
$5_{15}$ & 1.056 & 0.263 & 2.685 & -0.585 & -0.162 & 0.096 \\
$6_{06}$ & 0.631 & -0.001 & 1.763 & -0.006 & -0.127 & 0.073 \\
$6_{15}$ & 0.943 & 0.185 & 2.580 & -0.434 & -0.119 & 0.070 \\
$6_{16}$ & 0.888 & 0.184 & 1.546 & -0.442 & -0.118 & 0.065 \\
\hline \hline
\end{tabular}



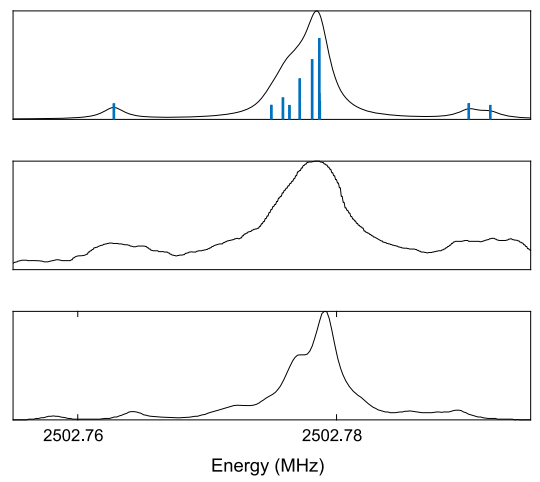

(a) $2_{11} A_{2} \leftarrow 2 A_{12} A_{1}$
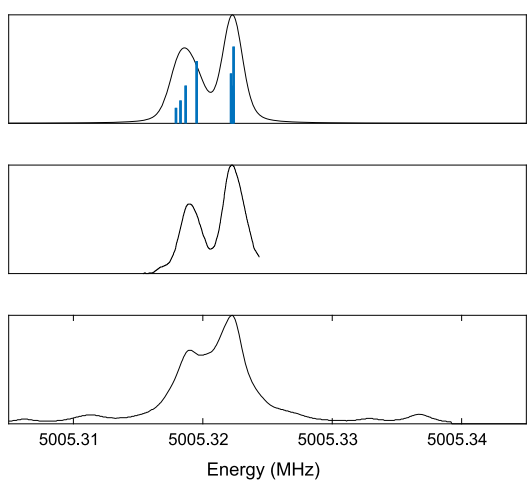

(b) $3_{12} A_{2} \leftarrow 3_{13} A_{1}$
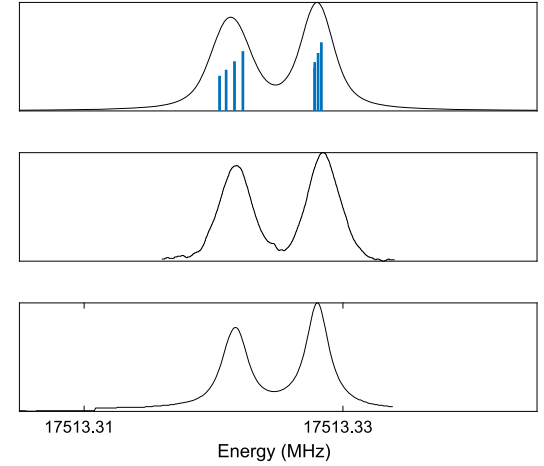

(c) $6_{15} A_{2} \leftarrow 6_{16} A_{1}$

FIG. 2. Comparison of our hyperfine spectra calculated (upper) for the $A$ levels with the measured spectra (middle) ${ }^{11}$ and the fit by Coudert et al. ${ }^{8}$ (bottom). The blue bars indicate the individual hyperfine transitions with a length proportional to the intensity. The hyperfine transitions are convoluted using a Voigt profile with $\sigma=0.11 \mathrm{kHz}$ and $\gamma=1.0 \mathrm{kHz}$ for the $2_{11} A_{2} \leftarrow 2{ }_{12} A_{1}$ transition, $\sigma=0.63 \mathrm{kHz}$ and $\gamma=0.3 \mathrm{kHz}$ for the $3_{12} A_{2} \leftarrow 3_{13} A_{1}$ transition, and $\sigma=0.767 \mathrm{kHz}$ and $\gamma=0.9 \mathrm{kHz}$ for the $6_{15} A_{2} \leftarrow 6_{16} A_{1}$ transition.

For all of the hyperfine transitions of $A$ symmetry, we observe good agreement between the calculated and measured hyperfine spectra. In the $2_{11} A_{2} \leftarrow 2_{12} A_{1}$ spectrum, we even reproduce the satellite peaks at the correct frequencies, and also find an asymmetric central peak. Also Coudert et al. reproduce these transitions very well in their fits. For the $3_{12} A_{2} \leftarrow 3_{13} A_{1}$ transition our results seem to be slightly better because the two peaks are more separated, just as in the experimental spectrum.

For the transitions of $E$ symmetry the agreement is slightly worse, but the resolution in the experimental spectra seems to be lower for these transitions. In the $2_{1} E \leftarrow 3_{0} E$ spectrum, the experiment shows a single peak with a satellite on the high-frequency side. Coudert $e t$ al. find a single peak in their simulated spectrum, while we find a satellite peak at higher frequency, although much stronger than seen in experiment. For the $4_{2} E \leftarrow 4_{1} E$ transition, the measured spectrum shows a strong peak with a side peak at about $40 \%$ of the intensity. Coudert $e t$ al. theoretically predict two equally strong peaks with similar intensity. We find a spread-out group of hyperfine lines, about as wide as the experimental spectrum. The experimental $5_{2} E \leftarrow 5_{1} E$ spectrum shows much noise and only a single peak could be resolved. In our simulations, we find the hyperfine transitions to be tightly grouped around the rotational frequency, which is compatible with the structure in the experimental spectrum. Coudert et al. compute two peaks within the width of the experimental peak.

In Fig. 4 we show some further $E$ symmetry transitions for which microwave spectra were measured both in Lille and in Hannover. ${ }^{8}$ The middle figures give both experimental spectra, with the spectra from Hannover and Lille depicted in red and blue, respectively. Since the spectra look different, we also simulated two spectra for each transition. In the two simulations, we used the same line frequencies and intensities calculated directly from our coupling parameters, but we applied a different Doppler shift in accordance with the measured shifts, and we used different parameters for the Voigt profile used in the convolution of our line spectra.

Comparing the measured spectra for the $2_{0} E \leftarrow 3_{-1} E$ transition, we observe that the Lille experiments show only a single peak with a small dip in the top, whereas the Hannover experiments show two peaks with the high frequency peak
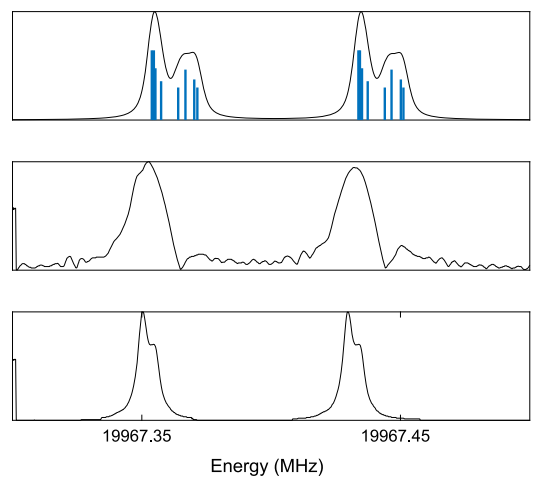

(a) $2_{1} E \leftarrow 3_{0} E$
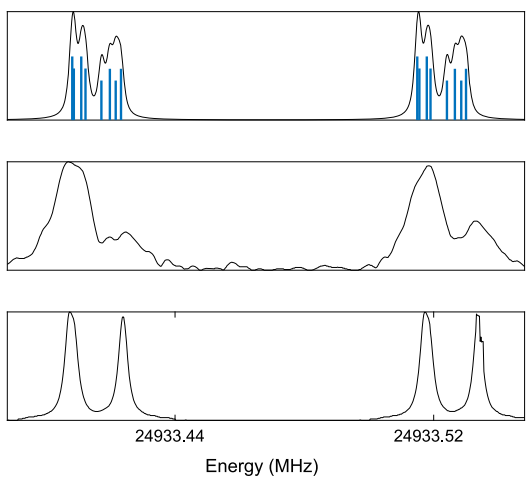

(b) $4_{2} E \leftarrow 4_{1} E$
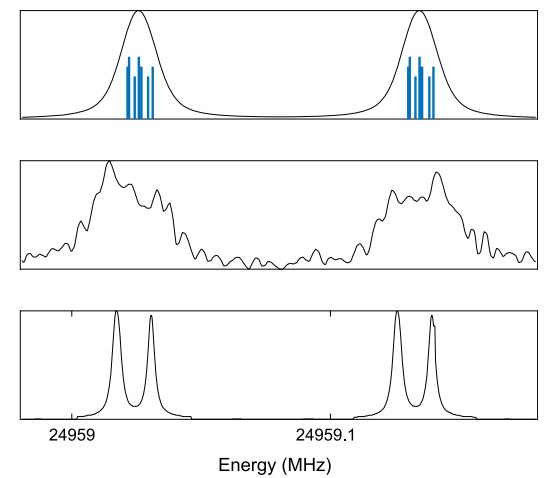

(c) $5_{2} E \leftarrow 5_{1} E$

FIG. 3. Comparison of our hyperfine spectra calculated (upper) for the $E$ levels with the measured spectra (middle) ${ }^{8}$ and the fit by Coudert $e t$ al. ${ }^{8}$ (bottom). The $2_{1} E-3_{0} E, 4_{2} E \leftarrow 4_{1} E$, and $8_{-2} E \leftarrow 9_{-1} E$ transitions are observed by Coudert et al. ${ }^{8}$ and have a Doppler splitting of $79.78 \mathrm{kHz}, 106.81 \mathrm{kHz}$, and $108.57 \mathrm{kHz}$. The blue bars indicate the individual hyperfine transitions with a length proportional to the intensity. The hyperfine transitions are convoluted using a Voigt profile with $\sigma=1.5 \mathrm{kHz}$ and $\gamma=1.88 \mathrm{kHz}$ for the $2_{1} E \leftarrow 3_{0} E$ transition, $\sigma=0.5 \mathrm{kHz}$ and $\gamma=0.88 \mathrm{kHz}$ for the $4_{2} E \leftarrow 4_{1} E$ transition, and $\sigma=3.5 \mathrm{kHz}$ and $\gamma=4.25 \mathrm{kHz}$ for the $5_{2} E \leftarrow 5_{1} E$ transition. 

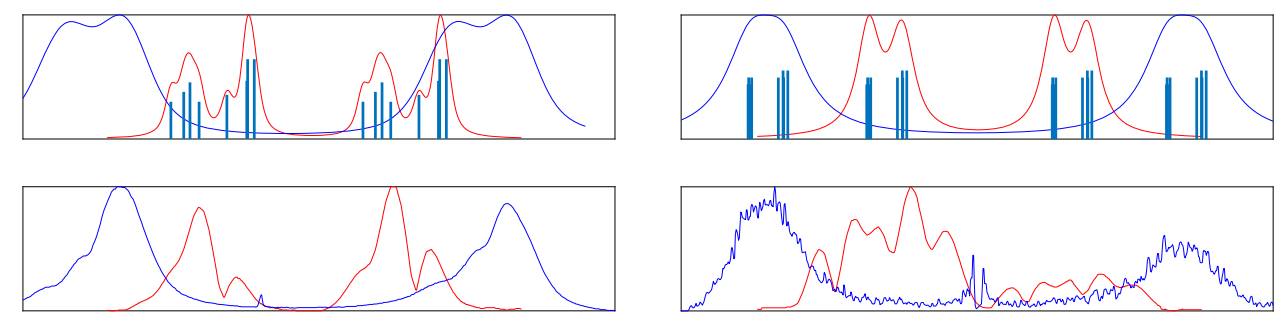

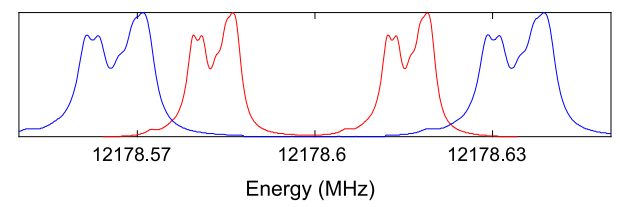

(a) $2_{0} E \leftarrow 3_{-1} E$

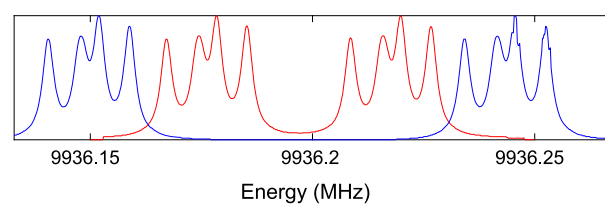

(b) $9_{-1} E \leftarrow 8_{-2} E$

FIG. 4. Comparison of our hyperfine spectra calculated (upper) for the $E$ levels with the measured spectra (middle) ${ }^{8}$ and the fit by Coudert $e t$ al. ${ }^{8}$ (bottom). The spectra were observed in Hannover (red) and Lille ${ }^{8}$ (blue). The blue bars indicate the individual hyperfine transitions with a length proportional to the intensity; for clarity they are only given for the $2_{0} E-3_{-1} E$ transition in our simulations of the Hannover spectrum. The Hannover spectra for the $2_{0} E \leftarrow 3_{-1} E$ and $9_{-1} E \leftarrow 8_{-2} E$ transitions are simulated by using a Voigt profile of $\sigma=0.5 \mathrm{kHz}$ and $\gamma=0.88$ and $2.88 \mathrm{kHz}$ and by using a Doppler splitting of 32.4 and $41.67 \mathrm{kHz}$. The Lille spectra for the $2_{0} E \leftarrow 3_{-1} E$ and $9_{-1} E \leftarrow 8_{-2} E$ transitions are simulated by using a Voigt profile with $\sigma=4.5$ and $0.5 \mathrm{kHz}$ and $\gamma=3.88$ and $6.50 \mathrm{kHz}$ and by using a Doppler splitting of 65.44 and $94.11 \mathrm{kHz}$.

being weaker. Comparing the two experimental spectra for the $9_{-1} E \leftarrow 8_{-2} E$ transition, we observe multiple peaks in the seemingly better resolved spectrum from Hannover, and only a single broad peak in the spectrum from Lille. However, the multiple peaks in the Hannover spectrum cover a wider range of frequencies than the single peak in the Lille spectrum, and there is a marked difference between the two Doppler components that should be nearly identical. This difference is most pronounced in the Hannover spectrum. So, it seems that there were some experimental problems in recording these spectra. Given these uncertainties in the experimental spectra, a detailed comparison with our calculated spectra is not very meaningful. All we can say is that the structure in our calculated spectra is probably compatible with the measured results.

In a recent paper of Belov et al. ${ }^{21}$ on Lamb-dip submillimeter-wave spectra of high- $J$ torsion-rotation transitions of $E$-symmetry in methanol, they found unexpectedly large doublet splittings in the spectra due to hyperfine coupling. They could explain these doublet splittings by a theoretical model that includes only the spin-rotation coupling within the $\mathrm{CH}_{3}$ subunit, with operators that depend on the torsion angle. The separation between the doublet peaks was observed for transitions between the rotational levels with $J$ ranging from 13 to 34. Extrapolation of the results to lower $J$ values with the aid of their model would yield a doublet separation of $17 \mathrm{kHz}$ in the $9_{-1} E \leftarrow 8_{-2} E$ spectrum. ${ }^{37}$ The middle panel of Fig. 4(b) shows, however, that such a doublet structure was not observed in the microwave spectra taken in Hannover and Lille. As shown in the upper panel of Fig. 4(b), our calculations with the full hyperfine coupling Hamiltonian including torsion-angle dependent spin-rotation, spin-torsion, and spin-spin interactions predict two sets of closely spaced lines. These lines, when not individually resolved, do give rise to a doublet structure, with a separation of about $7.5 \mathrm{kHz}$ between the two peaks.

A complete set of tables with our calculated transition frequencies and Einstein $A$-coefficients of all hyperfine transitions associated with the different rotational transitions discussed in the paper is given in the supplementary material.
Inspection of these tables shows clearly that for each rotational transition with given $\Delta J=0$ or \pm 1 only the hyperfine transitions with $\Delta F=\Delta J$ have substantial intensities, while the other hyperfine components are weaker by about an order of magnitude. So, the spectra are strongly dominated by these particular hyperfine transitions, which are shown as the line spectra in our figures.

Concluding this section, we note that with most of the dipole-dipole, spin-rotation, and spin-torsion coupling parameters from $a b$ initio calculations and only the electronic contribution to the dominant spin-torsion parameters obtained from a fit to two experimental spectra - one for an $A$ symmetry transition and one for an $E$ symmetry transition-we obtain good agreement with these spectra, as well as with another set of measured spectra not used in the fit. One should realize, however, that the individual hyperfine transitions were not resolved in the measured spectra, which limits the amount of experimental data that we could use in our fit. There may be a relatively large uncertainty in the fitted spin-torsion coupling parameters, which can only be reduced when more of the detailed hyperfine structure in the spectra that we predict will actually be resolved.

\section{DISCUSSION}

In order to understand the structure in the hyperfine spectra, it is useful to consider the nature of the hyperfine splittings of the torsion-rotation levels. Figures 5(a) and 5(b) illustrate these splittings for two torsion-rotation levels, one of $A$ symmetry and one of $E$ symmetry. The hyperfine levels were computed with the parameters listed in Tables I and II. The middle column in these figures shows the effect of the couplings involving the $\mathrm{CH}_{3}$ protons, the righthand column shows the additional splittings when also the interactions with the $\mathrm{OH}$ proton are included. The intermediate quantum number $F_{1}$ is obtained by coupling the overall rotation angular momentum $J$ with the collective nuclear spin $I_{123}$ of the three $\mathrm{CH}_{3}$ protons. We mentioned above that $I_{123}=3 / 2$ for $A$ symmetry states, 


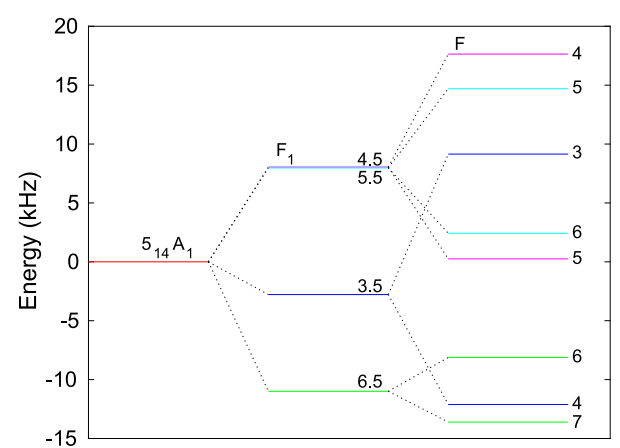

(a) Splitting of torsion-rotation level $5_{14} A_{1}$

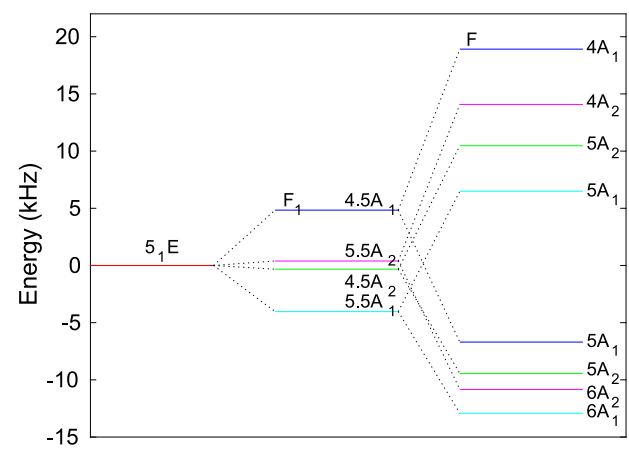

(b) Splitting of torsion-rotation level $5_{1} E$.

FIG. 5. Hyperfine splitting of torsion-rotation $A$ and $E$ levels. The middle column shows the effects of the interactions involving the $\mathrm{CH}_{3}$-protons, with the intermediate quantum number $F_{1}$ explained in the text, the righthand column the final hyperfine levels labeled with the quantum number $F$.

so that for $J \geq 2$ we obtain four different $F_{1}$ values ranging from $J-3 / 2$ to $J+3 / 2$. The additional coupling with the spin $I_{4}=1 / 2$ of the $\mathrm{OH}$ proton then yields five different $F$ values ranging from $J-2$ to $J+2$, with the intermediate values of $F$ occurring twice and the largest and smallest $F$ value only once. For states of $E$ symmetry $I_{123}=1 / 2$, so that only two different values of $F_{1}$ are obtained. Additional coupling with $I_{4}=1 / 2$ then yields three $F$ values: $J-1, J, J+1$, with two hyperfine states for $F=J$ and only a single one for $F=J \pm 1$. However, the irrep $E$ carries two spatial and two nuclear spin wave functions which may be combined to a total wave function of $A_{1}$ or $A_{2}$ symmetry, see Eq. (23) in Sec. II C. Each angular momentum coupling scheme then yields two Pauli-allowed states.

Since the distance between the $\mathrm{OH}$ proton and each of the $\mathrm{CH}_{3}$ protons is much larger than the distance between the protons within the $\mathrm{CH}_{3}$ group, one might expect that the largest splitting between the hyperfine levels originates from the interactions within the $\mathrm{CH}_{3}$ group, and that the coupling with the $\mathrm{OH}$ proton only causes further small splittings. The actual picture is more subtle, however. The magnetic dipole-dipole interactions are indeed stronger between the $\mathrm{CH}_{3}$ protons than between the $\mathrm{OH}$ proton and the $\mathrm{CH}_{3}$ protons. Tables I and IV show that the spin-rotation interactions are of the same magnitude for $\mathrm{CH}_{3}$ and $\mathrm{OH}$ protons. However, the spin-torsion interactions are stronger for the $\mathrm{OH}$ proton than for the $\mathrm{CH}_{3}$ protons, and they counteract the spin-rotation interactions. As a result we find for states of $A$ symmetry that the hyperfine splitting due to the $\mathrm{CH}_{3}$-protons is of the same magnitude as the additional splittings caused by the $\mathrm{OH}$ proton. For states of $E$ symmetry the interactions involving the $\mathrm{OH}$ proton dominate the hyperfine splittings.

An additional important factor that determines the structure of the hyperfine spectra is the transition line strengths. Transitions are allowed for $\Delta F=0, \pm 1$, but we find, in agreement with the experience from the experimental data, that the hyperfine components of a given rotational transition $\Delta J$ are considerably stronger for transitions with $\Delta F=\Delta J$ than for the other allowed transitions. Only those lines will be visible in the measured hyperfine spectra, which yield a considerable simplification of these spectra.

The expressions that we derived for the spin-rotation coupling tensors $\boldsymbol{M}_{K}$ and spin-torsion coupling tensors $\boldsymbol{d}_{K}$ differ from the expressions presented by Heuvel et al. ${ }^{10}$ and used also by Coudert et al. ${ }^{8}$ The main difference is that in the formulas of Heuvel et al. for these tensors a Thomas precession factor appears in the relative velocity vectors of the interacting particles. Furthermore, we found a sign difference in one of the terms in the hyperfine Hamiltonian. We checked the importance of such a Thomas correction factor in the calculations of the nuclear contributions to the coupling tensors and conclude that it has a substantial effect on the calculated results. Even more so, since the Thomas correction factor was written by Heuvel et al. as $\gamma_{K}=Z_{K} M_{p} /\left(g_{K} M_{K}\right)$ (for protons $\approx 0.18$ ), instead of $\gamma_{K}=1-Z_{K} M_{p} /\left(g_{K} M_{K}\right)$ (for protons $\left.\approx 0.82\right)$, as it should be ${ }^{15}$ Explicit derivations ${ }^{15,19,20}$ have shown, however, that the Thomas precession correction vanishes completely for molecules at equilibrium, and that it should be included in a different manner for molecules that deviate from their equilibrium geometry. In the present paper, we use $\gamma_{K}=1$ and obtain the Thomas correction to both the spin-rotation and spin-torsion coupling tensors with formulas that we derived specifically for molecules with large amplitude internal rotation, such as methanol. Application of these formulas shows that the Thomas corrections are negligibly small, so that also our numerical results differ substantially from those of Coudert et al. ${ }^{8}$

\section{CONCLUSION}

Expressions for the spin-rotation and spin-torsion coupling terms in the hyperfine Hamiltonian for molecules with internal rotation are derived from first principles. The spinrotation coupling Hamiltonian contains not only the usual term with the rotational angular momentum operator $\hat{\boldsymbol{J}}$-which is the spin-rotation coupling term in the hyperfine Hamiltonian for semi-rigid molecules-but also a term that contains the torsional angular momentum operator $\hat{p}_{\gamma}$. Vice-versa, the spintorsion coupling Hamiltonian-present only for molecules with internal rotation-contains not only a term with $\hat{p}_{\gamma}$ but also a term with $\hat{\boldsymbol{J}}$. This hyperfine Hamiltonian was derived previously by Heuvel and Dymanus, ${ }^{10}$ but our result is essentially different from theirs especially in the way to treat the relativistic Thomas precession correction. In the Hamiltonian of Heuvel and Dymanus this correction was taken into account by putting a scaling factor into the relative velocity vectors of the interacting particles, which has a substantial effect on the 
calculated results. However, it was shown in a paper by Flygare $^{15}$ that the Thomas correction actually vanishes for the molecules at equilibrium and in some later papers on diatomic molecules ${ }^{18-20}$ that it is very small for the molecules that vibrate about their equilibrium geometry. Here, we derive a formula for the Thomas correction to the spin-rotation and spintorsion coupling in molecules with internal rotation. Application of this formula shows that also for methanol the Thomas correction is negligibly small, even though the molecule exerts an internal rotation far from equilibrium.

Most of the coupling parameters in the hyperfine Hamiltonian for methanol could be obtained from ab initio electronic structure calculations at the $\operatorname{CCSD}(\mathrm{T})$ level with the program CFOUR. ${ }^{25}$ The electronic contribution to the spin-torsion coupling parameters could not be obtained in this manner, however. So we fitted the missing spin-torsion parameters by computations of the hyperfine levels and transition intensities for the $5_{15} A_{2} \leftarrow 6_{06} A_{1}$ and $3_{2} E \leftarrow 3_{1} E$ rotational transitions in methanol and compared with the measured spectra ${ }^{8}$ in which the hyperfine structure in the spectrum is partly resolved. With the obtained hyperfine parameters, we calculated the hyperfine structure in the spectra for several other rotational transitions between the torsional states of both $A$ and $E$ symmetry, and find that also they agree well with the measured spectra of Heuvel and Dymanus ${ }^{11}$ and Coudert et al. ${ }^{8}$ In contrast with the work of Coudert et al. ${ }^{8}$ where the dominant spin-rotation coupling parameters were included in the fit and were found to deviate substantially from the $a b$ initio calculated values, we kept the $a b$ initio values and did not include these parameters in our fit. Another difference between our work and Ref. 8 is that in our case the fitted electronic contributions to the spin-torsion coupling parameters partly cancel the nuclear contributions, i.e., they have opposite signs as expected, whereas it was found in Ref. 8 that these contributions are about equal in magnitude and have the same sign, which seems unphysical.

Currently we are using our results obtained for the hyperfine Hamiltonian of methanol in calculations of the Zeeman splittings of the hyperfine transitions by external magnetic fields. Quantitative information on these magnetic field effects is relevant for the analysis of astrophysical data, such as the methanol maser spectra from star-forming regions. This work will be reported in a forthcoming publication.

\section{SUPPLEMENTARY MATERIAL}

See supplementary material for figures of other spectra analyzed and tables with all calculated hyperfine transition frequencies and intensities.

\section{ACKNOWLEDGMENTS}

We thank Li-Hong Xu for providing the source code of her extended version of the program BELGI, and Laurent Coudert and Jens-Uwe Grabow for making available their results prior to publication. We are grateful to Jon Hougen for reading our manuscript and for valuable comments. We acknowledge useful discussions with Huib Jan van Langevelde, Wouter Vlemmings, and Gabriele Surcis. This work was partially supported by the Swedish Research Council (VR).

\section{APPENDIX A: THEORY}

\section{Kinetic energy operator}

As usual for molecules with internal rotation, we distinguish two parts in the molecule that are internally rigid. The "frame" part consists of those nuclei that remain fixed in the molecule-fixed (MF) principal axis frame, in the case of methanol the $\mathrm{OH}$ fragment. The origin of the MF frame is the center of mass of the $\mathrm{CH}_{3} \mathrm{OH}$ molecule and its orientation with respect to a space-fixed (SF) frame is defined by three $z y z$-Euler angles $(\chi, \theta, \phi)$. The "top" part is fixed with respect to a frame TF that rotates with respect to the MF frame about a single (fixed) axis $\lambda$ over an angle $\gamma$. This "top" is considered to be a symmetric rotor, such as the $\mathrm{CH}_{3}$ group in methanol. The coordinates of the nuclei in the "frame" and "top" with respect to the SF frame are given by

$$
\begin{aligned}
\boldsymbol{r}_{K}^{\mathrm{frame}, \mathrm{SF}} & =\boldsymbol{R}^{-1}(\chi, \theta, \phi) \boldsymbol{r}_{K}^{\mathrm{frame}, \mathrm{MF}}, \\
\boldsymbol{r}_{K}^{\mathrm{top}, \mathrm{SF}} & =\boldsymbol{R}^{-1}(\chi, \theta, \phi) \boldsymbol{R}^{-1}(\lambda, \gamma) \boldsymbol{r}_{K}^{\mathrm{top}, \mathrm{TF}},
\end{aligned}
$$

where $\boldsymbol{R}(\chi, \theta, \phi)$ is a rotation over the $z y z$-Euler angles and $\boldsymbol{R}(\lambda, \gamma)$ is a rotation over $\gamma$ about the axis $\lambda$, and the nuclear coordinates $\boldsymbol{r}_{K}^{\text {frame,MF }}$ and $\boldsymbol{r}_{K}^{\text {top,TF }}$ are fixed with respect to their respective frames. The time-derivatives of the SF nuclear positions are related to the time-independent $\mathrm{MF}$ and $\mathrm{TF}$ atomic coordinates

$$
\begin{aligned}
\dot{\boldsymbol{r}}_{K}^{\mathrm{frame}, \mathrm{SF}}= & \dot{\boldsymbol{R}}^{-1}(\chi, \theta, \phi) \boldsymbol{r}_{K}^{\mathrm{frame}, \mathrm{MF}} \\
\dot{\boldsymbol{r}}_{K}^{\mathrm{top}, \mathrm{SF}}= & \dot{\boldsymbol{R}}^{-1}(\chi, \theta, \phi) \boldsymbol{R}^{-1}(\lambda, \gamma) \boldsymbol{r}_{K}^{\mathrm{top}, \mathrm{TF}} \\
& +\boldsymbol{R}^{-1}(\chi, \theta, \phi) \dot{\boldsymbol{R}}^{-1}(\lambda, \gamma) \boldsymbol{r}_{K}^{\mathrm{top}, \mathrm{TF}}
\end{aligned}
$$

The SF derivatives, transformed to the MF frame for the nuclei in the "frame" and to the TF frame for the nuclei in the "top," can also be expressed as

$$
\begin{gathered}
\boldsymbol{R}(\chi, \theta, \phi) \dot{\boldsymbol{r}}_{K}^{\mathrm{frame}, \mathrm{SF}}=\omega \times \boldsymbol{r}_{K}^{\mathrm{frame}, \mathrm{MF}}, \\
\boldsymbol{R}(\lambda, \gamma) \boldsymbol{R}(\chi, \theta, \phi) \dot{\boldsymbol{r}}_{K}^{\mathrm{top}, \mathrm{SF}}=\omega^{\prime} \times \boldsymbol{r}_{K}^{\mathrm{top}, \mathrm{TF}}+\dot{\gamma} \lambda \times \boldsymbol{r}_{K}^{\mathrm{top}, \mathrm{TF}},
\end{gathered}
$$

in terms of the angular velocities $\omega, \dot{\gamma}$, and $\omega^{\prime}=\boldsymbol{R}(\lambda, \gamma) \omega$. The Lagrangian form of the classical kinetic energy in terms of the atomic masses $m_{K}$ and velocities $\dot{\boldsymbol{r}}_{K}^{\mathrm{SF}}$ can then be rewritten as

$$
2 T=\boldsymbol{\omega}^{T} \boldsymbol{I} \boldsymbol{\omega}+\dot{\gamma} \lambda^{T} \boldsymbol{I}^{\mathrm{top}} \boldsymbol{\omega}+\dot{\gamma} \boldsymbol{\omega}^{T} \boldsymbol{I}^{\mathrm{top}} \boldsymbol{\lambda}+I_{\gamma} \dot{\gamma}^{2},
$$

in which $\boldsymbol{I}=\boldsymbol{I}^{\text {frame }}+\boldsymbol{I}^{\text {top }}$ is the overall inertia tensor and $I_{\gamma}=\lambda^{T} \boldsymbol{I}^{\text {top }} \lambda$ is the moment of inertia of the top about the internal rotation axis $\lambda$. Since the "top" is a symmetric rotor, its inertia tensor is not changed by the internal rotation, $\boldsymbol{I}^{\text {top }}=\boldsymbol{R}^{-1}(\lambda, \gamma) \boldsymbol{I}^{\text {top }} \boldsymbol{R}(\lambda, \gamma)$. Also the overall inertia tensor $\boldsymbol{I}$, diagonal in the principal axes frame MF, is therefore invariant under the internal rotation. In Eq. (A4) the components of the vectors $\boldsymbol{\omega}$ and $\lambda$ and of the inertia tensors $\boldsymbol{I}$ and $\boldsymbol{I}^{\text {top }}$ are given relative to the MF frame.

This equation can be written in matrix form as

$$
2 T=\left(\begin{array}{c}
\omega \\
\dot{\gamma}
\end{array}\right)^{T}\left(\begin{array}{cc}
\boldsymbol{I} & \boldsymbol{I}^{\mathrm{top}} \boldsymbol{\lambda} \\
\boldsymbol{\lambda}^{T} \boldsymbol{I}^{\mathrm{top}} & I_{\gamma}
\end{array}\right)\left(\begin{array}{c}
\omega \\
\dot{\gamma}
\end{array}\right) .
$$

In order to derive the quantum mechanical expression for $T$, one has to convert the Lagrangian in Eq. (A5) into the 
corresponding classical Hamiltonian with the angular momenta $\boldsymbol{J}$ and $p_{\gamma}$ conjugate to the angular velocities $\boldsymbol{\omega}$ and $\dot{\gamma}$ given by

$$
\begin{aligned}
& \boldsymbol{J}=\frac{\partial T}{\partial \boldsymbol{\omega}}=\boldsymbol{I} \boldsymbol{\omega}+\boldsymbol{I}^{\mathrm{top}} \boldsymbol{\lambda} \dot{\gamma} \\
& p_{\gamma}=\frac{\partial T}{\partial \dot{\gamma}}=\boldsymbol{\omega} \cdot \boldsymbol{I}^{\mathrm{top}} \boldsymbol{\lambda}+I_{\gamma} \dot{\gamma}
\end{aligned}
$$

After inversion of the $4 \times 4$ matrix in Eq. (A5) with the use of a formula in Ref. 38, the angular velocities are expressed in terms of their conjugate momenta

$$
\begin{aligned}
& \boldsymbol{\omega}=\boldsymbol{I}^{-1} \boldsymbol{J}-2 F\left(p_{\gamma}-\boldsymbol{\rho} \cdot \boldsymbol{J}\right) \boldsymbol{\rho}, \\
& \dot{\gamma}=2 F\left(p_{\gamma}-\boldsymbol{\rho} \cdot \boldsymbol{J}\right),
\end{aligned}
$$

with

$$
F=\frac{1}{2}\left(I_{\gamma}-\boldsymbol{\rho}^{T} \boldsymbol{I} \boldsymbol{\rho}\right)^{-1} \quad \text { and } \quad \boldsymbol{\rho}=\boldsymbol{I}^{-1} \boldsymbol{I}^{\mathrm{top}} \lambda .
$$

When these results are substituted into Eq. (A5), the Hamiltonian kinetic energy becomes

$$
T=\frac{1}{2} \boldsymbol{J} \cdot \boldsymbol{I}^{-1} \boldsymbol{J}+F\left(p_{\gamma}-\boldsymbol{\rho} \cdot \boldsymbol{J}\right)^{2} .
$$

The quantum mechanical equivalent of this result, given by Hougen et al., ${ }^{22}$ is obtained by replacing the total angular momentum $\boldsymbol{J}$ by the operator $\hat{\boldsymbol{J}}^{26}$ and $p_{\gamma}$ by $(\hbar / i) \partial / \partial \gamma$. The components of $\hat{\boldsymbol{J}}$ are defined relative to the MF frame and obey the anomalous commutation relations that hold for body-fixed angular momentum operators. ${ }^{26}$

\section{Spin-rotation and spin-torsion interactions}

The magnetic field produced at position $\boldsymbol{r}_{K}$ by a set of moving particles $i$ (nuclei and electrons) with charges $Z_{i} e$ is given by

$$
\boldsymbol{B}_{K}=\sum_{i} \frac{Z_{i} e}{c} r_{K i}^{-3} \boldsymbol{r}_{K i} \times \boldsymbol{v}_{K i} .
$$

The vectors $\boldsymbol{r}_{K i}=\boldsymbol{r}_{i}-\boldsymbol{r}_{K}$ are the relative position vectors of the particles, $r_{K i}$ is the length of $\boldsymbol{r}_{K i}$, and $\boldsymbol{v}_{K i}=\dot{\boldsymbol{r}}_{K i}$ are their relative velocities. The magnetic moment of nucleus $K$ is $\boldsymbol{\mu}_{K}$ $=g_{K} \mu_{N} \boldsymbol{I}_{K}$, with $\boldsymbol{I}_{K}$ denoting the nuclear spin, $\mu_{N}=e /\left(2 M_{p} c\right)$ the nuclear magneton with the proton mass $M_{p}$ and the speed of light $c$, and $g_{K}$ the gyromagnetic factor of nucleus $K$. Its energy in the field $\boldsymbol{B}_{K}$ is ${ }^{17}$

$H_{K}=-\boldsymbol{B}_{K} \cdot \boldsymbol{\mu}_{K}=-\sum_{i} \frac{Z_{i} e g_{K} \mu_{N}}{c} r_{K i}^{-3}\left[\boldsymbol{r}_{K i} \times \boldsymbol{v}_{K i}\right] \cdot \boldsymbol{I}_{K}$.

In several papers ${ }^{10,14-16}$ one takes into account the Thomas precession factor $\gamma_{K}$ and replaces the vector $\boldsymbol{v}_{K i}=\boldsymbol{v}_{i}-\boldsymbol{v}_{K}$ by $\boldsymbol{v}_{i}-\gamma_{K} \boldsymbol{v}_{K}$. The correct expression for this factor is ${ }^{15}$

$$
\gamma_{K}=1-\frac{Z_{K} M_{p}}{g_{K} M_{K}}
$$

but different expressions occur in Refs. 10 and 14. Flygare $^{15}$ has shown that for the nuclear spin-rotation coupling in molecules at their equilibrium geometry, the Thomas precession correction vanishes. We omit this correction by using Eq. (A11) without any correction, i.e., by setting $\gamma_{K}=1$. In Sec. II B we have shown how it actually has to be dealt with in molecules with internal rotation such as methanol.
The positions of the nuclei in the "frame" and "top" of the molecule are fixed with respect to the MF and TF frame, respectively. Their positions with respect to the SF frame are given by Eq. (A1), and in Eq. (A3) their velocities are expressed in terms of the angular velocity $\omega$ of the whole molecule and the velocity $\dot{\gamma}$ of rotation of the "top" about the axis $\lambda$ relative to the "frame." Substitution of these expressions into Eq. (A11) yields

$$
H_{K}^{\text {frame }}=-\boldsymbol{I}_{K} \cdot \boldsymbol{W}_{K}^{\text {frame }} \boldsymbol{\omega}
$$

for the particles $i$ in the "frame," with

$$
\boldsymbol{W}_{K}^{\mathrm{frame}}=\sum_{i}^{\text {frame }} \frac{Z_{i} e g_{K} \mu_{N}}{c} r_{K i}^{-3}\left[\left(\boldsymbol{r}_{K i}^{\mathrm{MF}} \cdot \boldsymbol{r}_{K i}^{\mathrm{MF}}\right) \mathbf{1}-\boldsymbol{r}_{K i}^{\mathrm{MF}} \otimes \boldsymbol{r}_{K i}^{\mathrm{MF}}\right]
$$

For the particles in the "top" we get

$$
H_{K}^{\text {top }}=-\boldsymbol{I}_{K} \cdot \boldsymbol{W}_{K}^{\mathrm{top}} \boldsymbol{\omega}^{\prime}-\boldsymbol{I}_{K} \cdot \boldsymbol{w}_{K} \dot{\gamma},
$$

with

$$
\boldsymbol{W}_{K}^{\mathrm{top}}=\sum_{i}^{\text {top }} \frac{Z_{i} e g_{K} \mu_{N}}{c} r_{K i}^{-3}\left[\left(\boldsymbol{r}_{K i}^{\mathrm{TF}} \cdot \boldsymbol{r}_{K i}^{\mathrm{TF}}\right) \mathbf{1}-\boldsymbol{r}_{K i}^{\mathrm{TF}} \otimes \boldsymbol{r}_{K i}^{\mathrm{TF}}\right]
$$

and

$$
\boldsymbol{w}_{K}=\sum_{i}^{\text {top }} \frac{Z_{i} e g_{K} \mu_{N}}{c} r_{K i}^{-3}\left[\left(\boldsymbol{r}_{K i}^{\mathrm{TF}} \cdot \boldsymbol{r}_{K i}^{\mathrm{TF}}\right) \mathbf{1}-\boldsymbol{r}_{K i}^{\mathrm{TF}} \otimes \boldsymbol{r}_{K i}^{\mathrm{TF}}\right] \lambda .
$$

All tensor and vector components in Eqs. (A15)-(A17) are defined in the TF frame, while Eqs. (A13) and (A14) are written in the MF frame. We prefer to write all equations in the MF frame, i.e., the principal axes frame of the whole molecule. This can be achieved by transformation to the $\gamma$ dependent coupling tensor $\boldsymbol{W}_{K}^{\text {top }}(\gamma)=\boldsymbol{R}(\lambda, \gamma)^{-1} \boldsymbol{W}_{K}^{\text {top }} \boldsymbol{R}(\lambda, \gamma)$ and coupling vector $\boldsymbol{w}_{K}(\gamma)=\boldsymbol{R}(\lambda, \gamma)^{-1} \boldsymbol{w}_{K}$. Equation (A15) then becomes

$$
H_{K}^{\mathrm{top}}=-\boldsymbol{I}_{K} \cdot \boldsymbol{W}_{K}^{\mathrm{top}}(\gamma) \boldsymbol{\omega}-\boldsymbol{I}_{K} \cdot \boldsymbol{w}_{K}(\gamma) \dot{\gamma},
$$

in which also the components of $\boldsymbol{I}_{K}$ and $\boldsymbol{\omega}$ are given in the MF frame. If nucleus $K$ is in the "top," the position vector $\boldsymbol{r}_{K}$ depends on $\gamma$, and so does the coupling tensor $\boldsymbol{W}_{K}^{\text {frame }}$. Since all tensors and vectors are now given in the MF frame, we may add Eqs. (A13) and (A18) to obtain

$$
H_{K}=-\boldsymbol{I}_{K} \cdot \boldsymbol{W}_{K}(\gamma) \boldsymbol{\omega}-\boldsymbol{I}_{K} \cdot \boldsymbol{w}_{K}(\gamma) \dot{\gamma},
$$

with

$$
\begin{aligned}
\boldsymbol{W}_{K}(\gamma) & =\boldsymbol{W}_{K}^{\text {frame }}+\boldsymbol{W}_{K}^{\mathrm{top}}(\gamma) \\
& =\sum_{i} \frac{Z_{i} e g_{K} \mu_{N}}{c} r_{K i}^{-3}\left[\left(\boldsymbol{r}_{K i}^{\mathrm{MF}} \cdot \boldsymbol{r}_{K i}^{\mathrm{MF}}\right) \mathbf{1}-\boldsymbol{r}_{K i}^{\mathrm{MF}} \otimes \boldsymbol{r}_{K i}^{\mathrm{MF}}\right],
\end{aligned}
$$

where we note that the sum over particles $i$ now runs over all electrons and all nuclei other than $K$ in the whole molecule.

Next we replace the angular velocities $\omega$ and $\dot{\gamma}$ in Eq. (A20) by their conjugate angular momenta $\boldsymbol{J}$ and $p_{\gamma}$, with the use of Eq. (A7). With the vector $\rho$ given by Eq. (A8) and replacing the angular momenta $\boldsymbol{J}, p_{\gamma}$, and $\boldsymbol{I}_{K}$ by the corresponding operators, this yields the spin-rotation coupling Hamiltonian in Eq. (3) and the spin-torsion coupling Hamiltonian in Eq. (4).

Finally we note that the coupling tensors given in Eqs. (A17) and (A20) depend on the coordinates of both nuclei 
and electrons. The contributions that contain the nuclear coordinates can be directly calculated, since the positions of the nuclei are fixed with respect to the MF frame (for the nuclei in the "frame") or depend only on the torsion angle $\gamma$ (for the nuclei in the "top"). For the spin-rotation coupling tensors $\boldsymbol{M}_{K}$, it was shown that the electronic contributions can be obtained from second order perturbation theory ${ }^{15}$ with matrix elements over the molecule's ground state and excited electronic wave functions. Calculation of these spin-rotation coupling tensors has been implemented in the electronic structure program package CFOUR, ${ }^{25}$ and they can thus be computed as a function of $\gamma$. No such implementation yet exists, however, for the spin-torsion coupling tensors $\boldsymbol{w}_{K}$. Just as in the recent paper of Coudert et al., ${ }^{8}$ we calculate the nuclear contributions to the tensors $w_{K}$ and estimate the electronic contributions from a fit to experimental hyperfine spectra. The notations $\boldsymbol{M}_{K}$ and $\boldsymbol{w}_{K}$ for these tensors are used in the main body of this paper for the total nuclear and electronic contributions, unless we specify explicitly the contribution that is meant.

\section{APPENDIX B: ANGULAR MOMENTUM ALGEBRA OF HYPERFINE COUPLING TERMS}

A vast amount of literature, summarized by Eshbach and Strandberg ${ }^{39}$ and Bowater et al., ${ }^{40}$ exists on the matrix elements of hyperfine and Zeeman interactions. In these papers the rotational matrix elements are given in terms of rather cumbersome matrix elements of direction cosines and rotational angular momentum operators. A more general formalism, exemplified here for the angular momentum algebra of the magnetic dipole-dipole and nuclear-spin rotation coupling operators, can be written with spherical tensor operators. The nuclear-spin rotation interaction Hamiltonian for a molecule with nuclei $\alpha$ can be written in SF spherical components as the sum over terms

$$
-\hat{\boldsymbol{I}}_{\alpha} \cdot \boldsymbol{M}_{\alpha} \hat{\boldsymbol{J}}=\sum_{m m^{\prime}}(-1)^{m}\left(\hat{\boldsymbol{I}}_{\alpha}\right)_{-m}^{\mathrm{SF}}\left(\boldsymbol{M}_{\alpha}\right)_{m m^{\prime}}^{\mathrm{SF}} \hat{J}_{m^{\prime}}^{\mathrm{SF}}
$$

The coupling tensor $\boldsymbol{M}_{\alpha}$ is calculated in the molecular-axes frame MF and can be transformed to the SF frame by the rotation

$$
\left(\boldsymbol{M}_{\alpha}\right)_{m m^{\prime}}^{\mathrm{SF}}=\sum_{k k^{\prime}} D_{m k}^{(1) *}(\chi, \theta, \phi)\left(\boldsymbol{M}_{\alpha}\right)_{k k^{\prime}}^{\mathrm{MF}} D_{m^{\prime} k^{\prime}}^{(1)}(\chi, \theta, \phi) .
$$

We expand the product of the two Wigner $D$-matrix elements in irreducible components $D_{P Q}^{(L) *}(\chi, \theta, \phi)$, with $L$ adopting the values $0,1,2$, and $Q=-L, \ldots, L$,

$$
\begin{aligned}
-\hat{\boldsymbol{I}}_{\alpha} \cdot \boldsymbol{M}_{\alpha} \hat{\boldsymbol{J}}= & \sum_{L P Q}(-1)^{P+L} \sum_{m m^{\prime}}\left(\hat{\boldsymbol{I}}_{\alpha}\right)_{-m}^{\mathrm{SF}} \hat{J}_{m^{\prime}}^{\mathrm{SF}} \\
& \times\left\langle 1-m 1 m^{\prime} \mid L-P\right\rangle D_{P Q}^{(L) *}(\chi, \theta, \phi) \\
& \times \sum_{k k^{\prime}}(-1)^{1-k^{\prime}}\left(\boldsymbol{M}_{\alpha}\right)_{k k^{\prime}}\left\langle 1 k 1-k^{\prime} \mid L Q\right\rangle,
\end{aligned}
$$

and define the rank- $L$ irreducible components of the coupling tensor as

$$
\left(\boldsymbol{M}_{\alpha}\right)_{L Q}=\sum_{k k^{\prime}}(-1)^{1-k^{\prime}}\left(\boldsymbol{M}_{\alpha}\right)_{k k^{\prime}}\left\langle 1 k 1-k^{\prime} \mid L Q\right\rangle .
$$

The quantity between angular brackets is a Clebsch-Gordan coefficient.
By evaluating the matrix element $\left\langle\left(k_{1}\right) j_{1} m_{1}\right|$ $D_{P Q}^{(L) *}(\chi, \theta, \phi)\left|\left(k_{2}\right) j_{2} m_{2}\right\rangle$ and using the completeness relation ${ }^{41}$ twice, we may express the Wigner $D$-matrix element as

$$
\begin{aligned}
D_{P Q}^{(L) *}(\chi, \theta, \phi)= & \sum_{k_{1} j_{1} k_{2} j_{2}} \frac{\left[j_{1}\right]^{1 / 2}\left[j_{2}\right]^{1 / 2}}{[L]^{1 / 2}}(-1)^{j_{1}-k_{1}} \\
& \times\left(\begin{array}{ccc}
j_{1} & L & j_{2} \\
-k_{1} & Q & k_{2}
\end{array}\right) \hat{T}_{P}^{(L)}\left(k_{1} j_{1} ; k_{2} j_{2}\right),
\end{aligned}
$$

with the rank- $L$ irreducible spherical tensor operator $\hat{\boldsymbol{T}}^{(L)}\left(j_{1} ; j_{2}\right)$ defined by its components ${ }^{41}$

$\hat{T}_{P}^{(L)}\left(j_{1} ; j_{2}\right)=\sum_{m_{1} m_{2}}\left|j_{1} m_{1}\right\rangle\left\langle j_{2} m_{2}\right|(-1)^{j_{1}-m_{1}}[L]^{1 / 2}\left(\begin{array}{ccc}j_{1} & L & j_{2} \\ -m_{1} & P & m_{2}\end{array}\right)$.

The quantities in round brackets are Wigner $3 j$ symbols and the square brackets are shorthand for $[j]=2 j+1$. Next we couple the two irreducible tensor operators $\hat{\boldsymbol{I}}_{\alpha}$ and $\hat{\boldsymbol{J}}$ with the use of the general relation $^{42}$

$$
\left[\hat{\boldsymbol{A}}^{\left(\ell_{1}\right)} \otimes \hat{\boldsymbol{B}}^{\left(\ell_{2}\right)}\right]_{q}^{(\ell)}=\sum_{m_{1}=-\ell_{1}}^{\ell_{1}} \sum_{m_{2}=-\ell_{2}}^{\ell_{2}} \hat{A}_{\ell_{1} m_{1}} \hat{B}_{\ell_{2} m_{2}}\left\langle\ell_{1} m_{1} \ell_{2} m_{2} \mid \ell q\right\rangle,
$$

and couple the unit spherical tensor operator in the Wigner $D$-matrix element to the coupled operator $\left[\hat{\boldsymbol{I}}_{\alpha} \otimes \hat{\boldsymbol{J}}^{(L)}\right.$ to find

$$
\begin{aligned}
-\hat{\boldsymbol{I}}_{\alpha} \cdot \boldsymbol{M}_{\alpha} \hat{\boldsymbol{J}}= & \sum_{j_{1} j_{2} k_{1} k_{2}}(-1)^{j_{1}-k_{1}}\left[j_{1}\right]^{1 / 2}\left[j_{2}\right]^{1 / 2} \\
& \times \sum_{L}\left[\hat{\boldsymbol{T}}^{(L)}\left(j_{1} ; j_{2}\right) \otimes\left[\hat{\boldsymbol{I}}_{\alpha} \otimes \hat{\boldsymbol{J}}\right]^{(L)}\right]_{0}^{(0)} \\
& \times \sum_{Q}\left(\begin{array}{cc}
j_{1} & L j_{2} \\
-k_{1} & Q k_{2}
\end{array}\right)\left(\boldsymbol{M}_{\alpha}\right)_{L Q} .
\end{aligned}
$$

Analogously, we obtain for the magnetic dipole-dipole interaction

$$
\begin{aligned}
\hat{\boldsymbol{I}}_{\alpha} \cdot \boldsymbol{D}_{\alpha \beta} \hat{\boldsymbol{I}}_{\beta}= & -\sum_{j_{1} j_{2} k_{1} k_{2}}(-1)^{j_{1}-k_{1}}\left[j_{1}\right]^{1 / 2}\left[j_{2}\right]^{1 / 2} \\
& \times \sum_{L}\left[\hat{\boldsymbol{T}}^{(L)}\left(j_{1} ; j_{2}\right) \otimes\left[\hat{\boldsymbol{I}}_{\alpha} \otimes \hat{\boldsymbol{I}}_{\beta}\right]^{(L)}\right]_{0}^{(0)} \\
& \times \sum_{Q}\left(\begin{array}{cc}
j_{1} & L j_{2} \\
-k_{1} & Q k_{2}
\end{array}\right)\left(\boldsymbol{D}_{\alpha \beta}\right)_{L Q} .
\end{aligned}
$$

The rotational and nuclear spin angular momentum operators can be written in terms of irreducible spherical tensor operators as

$$
\begin{gathered}
\hat{J}_{q}=\sum_{j} \sqrt{\frac{[j] j(j+1)}{3}} \hat{T}_{1 q}(j, j), \\
\left(\hat{\boldsymbol{I}}_{\alpha}\right)_{q}=\sum_{i_{\alpha}} \sqrt{\frac{\left[i_{\alpha}\right] i_{\alpha}\left(i_{\alpha}+1\right)}{3}} \hat{T}_{1 q}\left(i_{\alpha}, i_{\alpha}\right) .
\end{gathered}
$$

Finally, as an example, we evaluate the matrix elements of the operator in Eq. (B9) with $\alpha=1$ and $\beta=2$ between coupled basis functions $\left|\left[\left(\left[\left\{I_{1} I_{2}\right\} I_{12} I_{3}\right] I_{123} I_{4}\right) I J\right] F \quad M_{F}\right\rangle$ and $\left|\left[\left(\left[\left\{I_{1}^{\prime} I_{2}^{\prime}\right\} I_{12}^{\prime} I_{3}^{\prime}\right] I_{123}^{\prime} I_{4}^{\prime}\right) I^{\prime} J^{\prime}\right] F M_{F}\right\rangle$ to find 


$$
\begin{aligned}
\langle & {\left.\left.\left[\left(\left\{I_{1} I_{2}\right\} I_{12}^{\prime} I_{3}\right] I_{123}^{\prime} I_{4}\right) I^{\prime} J^{\prime}\right] F M_{F}\left|\left[\hat{\boldsymbol{T}}^{(L)}\left(j_{1} ; j_{2}\right) \otimes\left[\hat{\boldsymbol{I}}_{1} \otimes \hat{\boldsymbol{I}}_{2}\right]^{(L)}\right]_{0}^{(0)}\right|\left[\left(\left[\left\{I_{1} I_{2}\right\} I_{12} I_{3}\right] I_{123} I_{4}\right) I J\right] F M_{F}\right\rangle } \\
= & \left(I_{1}\left(I_{1}+1\right) I_{2}\left(I_{2}+1\right)\right)^{1 / 2}\left[I_{1}\right]^{1 / 2}\left[I_{2}\right]^{1 / 2}[L]^{1 / 2}\left[I_{12}^{\prime}\right]^{1 / 2}\left[I_{12}\right]^{1 / 2}\left[I_{123}^{\prime}\right]^{1 / 2}\left[I_{123}\right]^{1 / 2}\left[I^{\prime}\right]^{1 / 2}[I]^{1 / 2}(-1)^{I_{12}^{\prime}+I_{3}+I_{4}+J+F+I_{123}+I_{123}^{\prime}+I^{\prime}+I+L} \\
& \times\left\{\begin{array}{ccc}
I_{1} & I_{1} & 1 \\
I_{2} & I_{2} & 1 \\
I_{12}^{\prime} & I_{12} & L
\end{array}\right\}\left\{\begin{array}{ccc}
I_{12} & I_{12}^{\prime} & L \\
I_{123}^{\prime} & I_{123} & I_{3}
\end{array}\right\}\left\{\begin{array}{ccc}
I_{123} & I_{123}^{\prime} & L \\
I^{\prime} & I & I_{4}
\end{array}\right\}\left\{\begin{array}{ccc}
J^{\prime} & J & L \\
I & I^{\prime} & F
\end{array}\right\}
\end{aligned}
$$

where the quantities between the curly brackets are Wigner $9 j$ and $6 j$ symbols. Matrix elements of the spin-rotation coupling and other dipole-dipole coupling terms can be found analogously.

${ }^{1}$ W. H. T. Vlemmings, Astron. Astrophys. 484, 773 (2008).

${ }^{2}$ G. Surcis, W. H. T. Vlemmings, H. J. van Langevelde, B. Hutawarakorn Kramer, and L. H. Quiroga-Nuñez, Astron. Astrophys. 556, A73 (2013).

${ }^{3}$ G. Surcis, W. H. T. Vlemmings, H. J. van Langevelde, and B. Hutawarakorn Kramer, Astron. Astrophys. 541, A47 (2012).

${ }^{4}$ P. Jansen, L.-H. Xu, I. Kleiner, W. Ubachs, and H. L. Bethlem, Phys. Rev. Lett. 106, 100801 (2011).

${ }^{5}$ J. Bagdonaite, P. Jansen, C. Henkel, H. L. Bethlem, K. M. Menten, and W. Ubachs, Science 339, 46 (2013).

${ }^{6}$ J. Bagdonaite, M. Daprà, P. Jansen, H. L. Bethlem, W. Ubachs, S. Muller,

C. Henkel, and K. M. Menten, Phys. Rev. Lett. 111, 231101 (2013).

${ }^{7}$ N. Kanekar, W. Ubachs, K. M. Menten, J. Bagdonaite, A. Brunthaler, C. Henkel, S. Muller, H. L. Bethlem, and M. Daprà, Mon. Not. R. Astron. Soc.: Lett. 448, L104 (2015).

${ }^{8}$ L. Coudert, C. Gutlé, T. Huet, J.-U. Grabow, and S. Levshakov, J. Chem. Phys. 143, 044304 (2015).

${ }^{9}$ J. E. M. Heuvel, "Hyperfine structure in internal rotor molecules," Ph.D. thesis, Katholieke Universiteit Nijmegen, 1972.

${ }^{10}$ J. Heuvel and A. Dymanus, J. Mol. Spectrosc. 47, 363 (1973).

${ }^{11}$ J. Heuvel and A. Dymanus, J. Mol. Spectrosc. 45, 282 (1973).

12 J. T. Hougen, W. L. Meerts, and I. Ozier, J. Mol. Spectrosc. 146, 8 (1991).

${ }^{13}$ L. H. Thomas, Philos. Mag. 3, 1 (1927).

${ }^{14}$ G. R. Gunther-Mohr, C. H. Townes, and J. A. van Vleck, Phys. Rev. 94, 1191 (1954).

${ }^{15}$ W. H. Flygare, J. Chem. Phys. 41, 793 (1964).

${ }^{16}$ P. Thaddeus, L. Krisher, and J. Loubser, J. Chem. Phys. 40, 257 (1964).

${ }^{17}$ W. Flygare, Molecular Structure and Dynamics (Prentice-Hall, Englewood Cliffs, 1978).

${ }^{18}$ J. Gauss and D. Sundholm, Mol. Phys. 91, 449 (1997).

${ }^{19}$ R. V. Reid and A. H.-M. Chu, Phys. Rev. A 9, 609 (1974).

${ }^{20}$ T. K. Rebane and M. I. Volodicheva, Vestn. Leningr. Univ. 22, 55 (1974).
${ }^{21}$ S. P. Belov, G. Y. Golubiatnikov, A. V. Lapinov, E. A. Ilyushin, V. V. Alekseev, A. A. Mescheryakov, J. T. Hougen, and L.-H. Xu, J. Chem. Phys. 145, 024307 (2016).

${ }^{22}$ J. Hougen, I. Kleiner, and M. Godefroid, J. Mol. Spectrosc. 163, 559 (1994).

${ }^{23}$ C. C. Lin and J. D. Swalen, Rev. Mod. Phys. 31, 841 (1959).

${ }^{24}$ L.-H. Xu, J. Fisher, R. M. Lees, H. Y. Shi, J. T. Hougen, J. C. Pearson, B. J. Drouin, G. A. Blake, and R. Braakman, J. Mol. Spectrosc. 251, 305 (2008).

${ }^{25}$ J. Stanton, J. Gauss, M. E. Harding, and P. Szalay, crour, coupled-cluster techniques for computational chemistry, http://www.cfour.de.

${ }^{26}$ D. M. Brink and G. R. Satchler, Angular Momentum, 3rd ed. (Clarendon, Oxford, 1993).

${ }^{27}$ J. D. Jackson, Classical Electrodynamics, 3rd ed. (Wiley, New York, 1998).

${ }^{28}$ H. Longuet-Higgins, Mol. Phys. 6, 445 (1963).

${ }^{29}$ P. R. Bunker and P. Jensen, Molecular Symmetry and Spectroscopy (NRC Research Press, 1998).

${ }^{30}$ B. Kirtman, J. Chem. Phys. 37, 2516 (1962).

${ }^{31}$ R. M. Lees and J. G. Baker, J. Chem. Phys. 48, 5299 (1968).

${ }^{32}$ E. Herbst, J. Messer, F. C. D. Lucia, and P. Helminger, J. Mol. Spectrosc. 108, 42 (1984).

${ }^{33}$ T. H. Dunning, Jr., J. Chem. Phys. 90, 1007 (1989).

${ }^{34}$ J. T. Hougen, J. Chem. Phys. 57, 4207 (1972).

${ }^{35}$ L. H. Coudert, W. Caminati, M. Schnell, and J.-U. Grabow, J. Mol. Spectrosc. 242, 118 (2007).

${ }^{36}$ M. Tudorie, L. H. Coudert, T. R. Huet, D. Jegouso, and G. Sedes, J. Chem. Phys. 134, 074314 (2011).

${ }^{37}$ J. T. Hougen, private communication (2016).

${ }^{38}$ D. S. Bernstein, Matrix Mathematics: Theory, Facts, and Formulas (Princeton University Press, 2009).

${ }^{39}$ J. R. Eshbach and M. W. P. Strandberg, Phys. Rev. 85, 24 (1952).

${ }^{40}$ I. C. Bowater, J. M. Brown, and A. Carrington, Proc. R. Soc. A 333, 265 (1973).

${ }^{41}$ K. Blum, Density Matrix Theory and Applications, Physics of Atoms and Molecules (Plenum, New York, 1981).

${ }^{42}$ L. C. Biedenharn, J. D. Louck, and P. A. Carruthers, Angular Momentum in Quantum Physics: Theory and Application (Addison-Wesley Reading, MA, 1981). 